\title{
Rvb1/Rvb2 proteins couple transcription and translation during glucose starvation
}

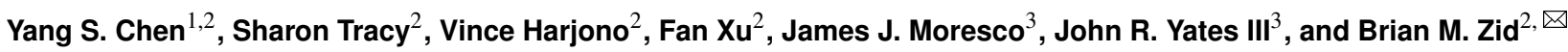 \\ ${ }^{1}$ Division of Biological Sciences, University of California, San Diego, La Jolla, CA 92093, USA \\ ${ }^{2}$ Department of Chemistry and Biochemistry, University of California San Diego, La Jolla, CA 92023, USA \\ ${ }^{3}$ Department of Chemical Physiology, The Scripps Research Institute, La Jolla, CA 92037, USA
}

\begin{abstract}
During times of unpredictable stress, organisms must adapt their gene expression to maximize survival. Along with changes in transcription, one conserved means of gene regulation during conditions that quickly represses translation is the formation of cytoplasmic phase-separated mRNP granules such as Pbodies and stress granules. Previously, we identified that distinct steps in gene expression can be coupled during glucose starvation as promoter sequences in the nucleus are able to direct the subcellular localization and translatability of mRNAs in the cytosol. Here, we report that Rvb1 and Rvb2, conserved ATPase proteins implicated as protein assembly chaperones and chromatin remodelers, were enriched at the promoters and mRNAs of genes involved in alternative glucose metabolism pathways that we previously found to be transcriptionally upregulated but translationally downregulated during glucose starvation in yeast. Engineered Rvb1/Rvb2-binding on mRNAs was sufficient to sequester the mRNAs into phase-separated granules and repress their translation. Additionally, this Rvb-tethering to the mRNA drove further transcriptional upregulation of the target genes. Overall, our results point to Rvb1/Rvb2 coupling transcription, mRNA granular localization, and translatability of mRNAs during glucose starvation. This Rvb-mediated rapid gene regulation could potentially serve as an efficient recovery plan for cells after stress removal.
\end{abstract}

mRNP granules | coupling in gene expression | mRNA localization | translational control

Correspondence: zid@ucsd.edu

Gene expression encompasses many steps across discrete cellular boundaries including transcription, mRNA processing and export, translation, and decay. Cells do not always live in stable and optimal conditions, instead they are faced with various types of stresses, such as nutrient starvation, heat shock, toxins, pathogens and osmotic stresses $(1,2)$. In dynamic environmental conditions, cells must balance disparate responses in gene expression as they quickly transition between homeostatic states. This can present challenges such as when cells repress overall translation while needing to upregulate the protein expression of stress response genes (3). To date, it is generally thought that mRNA cytoplasmic activities are predominantly dictated by cis-acting sequence elements within the RNA; however, coupling steps in gene expression presents an attractive strategy to overcome the challenges by creating regulons of mRNAs that are similarly controlled at the transcriptional level and can be coordinately tuned at the post-transcriptional level as well.

In recent years "imprinting" by co-transcriptional load- ing has been implicated as an alternative mechanism to cisacting RNA sequence elements in determining cytoplasmic mRNA fate (4). For instance, it was found that promoters determined mRNA decay rates through the co-transcriptional loading of RNA-binding proteins (RBPs) to the nascent RNA $(5,6)$. Similarly, Vera et al. showed that the translation elongation factor eEF1A coupled the transcription and translation of HSP70 mRNAs through co-transcriptional loading during heat shock in mammalian cells (7). Zander et al. showed that transcription factor Hsf1 might be functioning in loading the nuclear mRNA export protein Mex67 on stress-related mRNAs during heat shock in yeast (8).

During stressful conditions one proposed means of post-transcriptional control is the phase separation of select mRNA transcripts and post-transcriptional regulatory proteins into phase-dense, concentrated, and membrane-less cytoplasmic structures generally described as phase-separated granules $(9,10)$. Two well-known stress-induced phaseseparated messenger ribonucleoprotein (mRNP) granules are processing bodies (P-bodies) and stress granules $(11,12)$. During stress, the direct connection between the formation of these granules coincident with an overall translational reduction suggests that the localization of mRNAs to these cytoplasmic granules might sequester the mRNAs away from the translational machineries, thus repressing the translation of the mRNAs (13-16). Yet how mRNAs are partitioned to or excluded from stress-induced granules remains unclear.

Previously we found that during glucose starvation in yeast, promoter sequences play an important role in determining the cytoplasmic fate of mRNAs (10). mRNAs transcribed by active promoters in unstressed cells (Class III, e.g., PGK1, PAB1) were directed to P-bodies. Meanwhile, stress-induced mRNAs showed two distinct responses: $\mathrm{mR}$ NAs of most heat shock genes (Class I, e.g., HSP30, HSP26) are transcriptionally induced, actively translated, and remain diffuse in the cytoplasm; however, Class II mRNAs are transcriptionally induced but become sequestered in both Pbodies and stress granules and are associated with inactive translation. Class II mRNAs are enriched for alternative glucose metabolic function such as glycogen metabolism (e.g., GSY1, GLC3, GPH1). Surprisingly, instead of the mRNA sequence itself, the promoter sequence that sits in the nucleus directs the translation and cytoplasmic localization of the corresponding induced mRNAs. Specifically, Hsf1-target sequences were shown to direct mRNAs to be excluded from 
mRNP granules and well translated. However, the mechanism by which the promoter can couple steps of gene expression during glucose starvation is unclear. As the promoter exclusively resides in the nucleus, we hypothesize factors exist that interact with promoters and are co-transcriptionally loaded onto mRNA prior to nuclear export.

In this study, we developed a novel proteomics-based screening method that enabled us to identify Rvb1/Rvb2 as interacting proteins with the promoters of the Class II alternative glucose metabolism genes (e.g., GLC3) that are upregulated in transcription but downregulated in translation and have granular-localized mRNA transcripts. Rvb1/Rvb2 (known as RuvbL1/RuvbL2 in mammals) are two highly conserved AAA+ (ATPases Associated with various cellular Activities) proteins that are found in multiple nucleoprotein complexes. Structural studies have shown that they form a dodecamer comprised of a stacked Rvb1 hexametric ring and a Rvb2 hexametric ring. They were reported as the chaperones of multiprotein complexes involved in chromatin remodeling processes and other nuclear pathways including snoRNP assembly (17-23). These two proteins are generally thought to act on DNA but have been found to be core components of mammalian and yeast cytoplasmic stress granules (24). Rvb1/Rvb2 have also been shown to regulate the dynamics and size of stress granules $(25,26)$. The dual presence of Rvb1/Rvb2 at chromatin and stress granules hints to their potential in coupling activities in the nucleus and cytoplasm. Furthermore, a human homolog of Rvb2 was found to be an RNA-binding protein that promotes the degradation of translating HIV-1 GAG mRNA (27). Relatedly, in this study we found that Rvb1/Rvb2 have roles in coupling transcription, cytoplasmic mRNA localization, and translation of specific glucose starvation induced genes in yeast, providing insight into how gene expression can be coordinated during fluctuating environmental conditions.

\section{Results}

\section{Rvb1/Rvb2 are identified as potential co-transcriptionally loaded protein factors on the alternative glucose metabolism genes}

To identify proteins involved in the ability of promoter sequences to direct the cytoplasmic fate of mRNAs during stress, we developed Co-Transcriptional ImmunoPrecipitation (CoTrIP), a novel biochemical screening technique to identify co-transcriptionally loaded protein factors (Figure 1A). Here we modified a yeast plasmid containing LacObinding sites that was previously used as an efficient purification system to isolate histones $(28,29)$. To this plasmid we added a uniform open reading frame (ORF) and different promoters of interest. We then used FLAG-tagged LacI, which binds to the $\mathrm{LacO}$ sequences, and UV-crosslinking to purify the plasmid along with the nascent mRNAs, and co-transcriptionally loaded proteins. Thereafter, mass spectrometry was performed to identify proteins enriched in a promoter specific manner. Real-time quantitative PCR (RTqPCR) validates that the CoTrIP method yields enrichment of the target nascent mRNAs, indicating that proteins enriched could be co-transcriptionally loaded (Figure 1-figure supplement 1). Here, we performed CoTrIP of three plasmids (two heat shock genes' promoters, HSP30 and HSP26, and an alternative glucose metabolism gene's promoter, $G L C 3$ ) in cells subject to 10 minutes of glucose deprivation. Those promoters had previously been shown to be sufficient to determine the cytoplasmic fate of the uniform open reading frame (10).

After comparing the protein enrichment on GLC3 promoter and on HSP30/HSP26 promoters (Figure 1B, 1C), we were able to detect differences in protein factors across the specific classes of promoters. The ATP-dependent DNA RuvB-like helicase Rvb1 was enriched 10-fold more on GLC3 promoter plasmids versus both HSP30/HSP26 promoters (p-value $=0.02$ ). To further verify this enrichment, we compared our protein enrichment data against the CRAPome repository, a large database of contaminant proteins from various immunoprecipitation (IP) experiments, and we found that Rvb1 was significantly enriched on the GLC3 promotercontaining plasmid (Figure 1C) (30). Proteins that were both enriched in "promoter versus promoter comparison" as well as in comparison to the CRAPome are listed (Figure 1C).

Rvb1/Rvb2 are two highly conserved members of the $\mathrm{AAA}+$ family that are involved in multiple nuclear pathways (19). These two proteins are generally thought to act on DNA but have been found to be core components of mammalian and yeast cytoplasmic stress granules (24). Microscopy revealed that $\mathrm{Rvb} 1 / \mathrm{Rvb} 2$ are predominately present in the nucleus when cells are not stressed but a portion of them becomes localized to cytoplasmic granules that are not P-bodies in both 15-minute and 30-minute glucose starvation conditions (Figure 1-figure supplement 2). Rvb1/Rvb2's interactions with DNA in the nucleus and presence in the cytoplasm suggest the potential of $R v b 1 / R v b 2$ to shuttle between the nucleus and cytoplasm.

\section{Rvb1/Rvb2 are enriched at the promoters of endogenous al- ternative glucose metabolism genes}

To validate the CoTrIP results as well as more globally explore the location of Rvb1 and Rvb2 on DNA during stress, Chromatin ImmunoPrecipitation sequencing (ChIPseq) was used to investigate Rvb1/Rvb2's enrichment across the genome. Rvb1/Rvb2 were fused with a tandem affinity purification (TAP)-tag at the C-terminus and purified by rabbit IgG beads. The TAP-tagged strains grow at a normal rate ( 90-minute doubling time), which suggests TAPtagging does not generally disrupt the endogenous protein function of these essential proteins. Here, we performed ChIP-seq on Rvb1, Rvb2, and the negative control Pgk1 in 10 minutes of glucose starvation (Figure 2-figure supplement 3 , left). Rvb1/Rvb2 are enriched from the $-500 \mathrm{bp}$ to the transcription start site (TSS) along the genome at 10 minutes of glucose starvation, whereas ChIP-seq of the negative control Pgk1 is not enriched in the promoter region (Figure 2A). The overall enrichment on promoters is consistent with findings that Rvb's can function as chromatin remodelers (31). We 

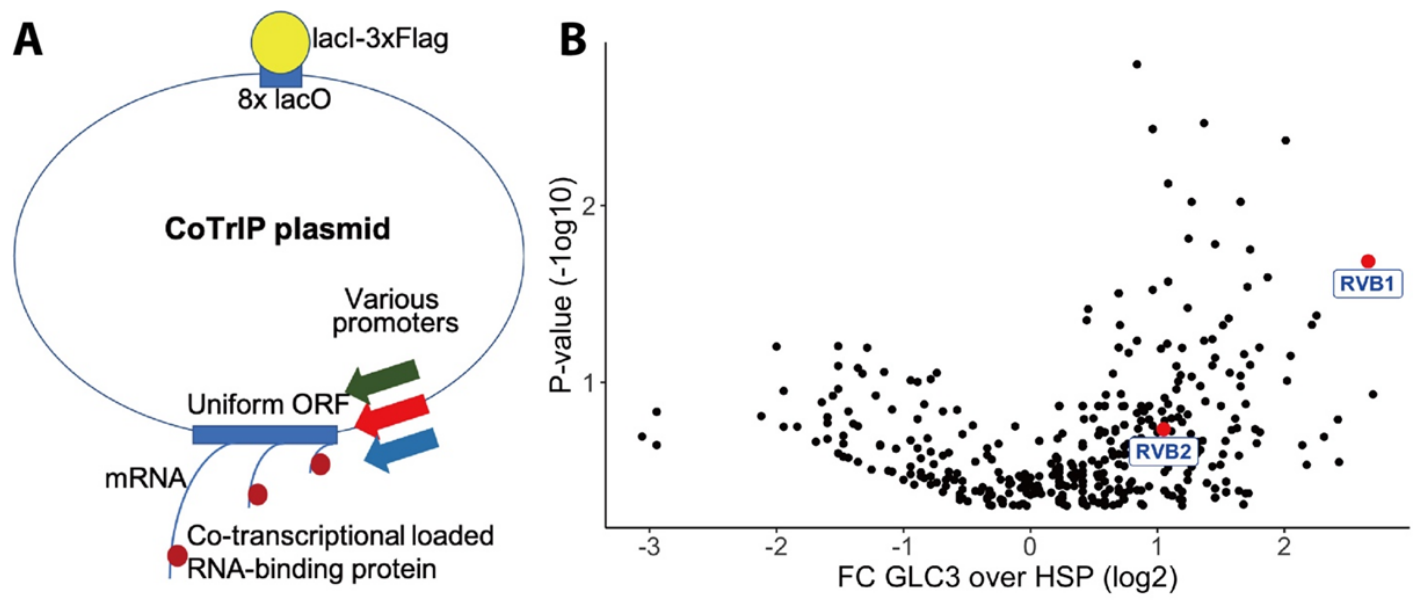

\begin{tabular}{llll}
\hline Protein factors & FC GLC3 over HSP & FC GLC3 over CRAPome & P-value \\
\hline Rvb1 & 6.32 & 20.71 & 0.021 \\
Rpp2a & 4.77 & 1.46 & 0.042 \\
Rps12 & 4.65 & 0.61 & 0.047 \\
Ste20 & 4.03 & 12.75 & 0.004 \\
Tef4 & 3.65 & 0.68 & 0.025 \\
Rpn3 & 3.32 & 6.45 & 0.018 \\
Hsp60 & 3.27 & 3.56 & 0.029 \\
Rpl18a; Rpl18b & 3.15 & 1.38 & 0.010
\end{tabular}

Fig. 1. Rvb1/Rvb2 are identified as potential co-transcriptional-loaded protein factors on alternative glucose metabolism genes. (A) A schematic view of CoTrlP. CoTrIP plasmid has an $8 \mathrm{X}$ lacO, a uniform ORF and various promoters of interest. CoTrIP plasmid was purified by immunoprecipitation of lacl-3XFlag protein. Enriched protein factors were identified by mass spectrometry. (B) Quantitative volcano plot of co-transcriptional-loaded protein candidates. X-axis: log2 scale of fold change of protein enrichment on 2 replicates of GLC3 promoter-containing CoTrIP plasmid over on 2 replicates of HSP30 promoter-containing and 1 replicate of HSP26 promoter-containing plasmid. Y-axis: minus log10 scale of the p-values from 2-sample t-test. Null hypothesis: enrichment on GLC3 promoter equals the enrichment on HSP promoters. Rvb1 and Rvb2 are highlighted in red dots and labeled. (C) Table of protein factors enriched on GLC3 promoters. FC of GLC3 vs HSP: fold change of protein enrichment on 2 replicates of GLC3 promoter-containing CoTrIP plasmid over on 2 replicates of HSP3O promoter-containing and 1 replicate of HSP26 promoter-containing plasmid. FC of GLC3 vs CRAPome: fold change of protein enrichment on 2 replicates of GLC3 promoter-containing CoTrIP plasmid over the CRAPome repository. CRAPome: a contaminant repository for affinity purification-mass spectrometry data. CRAPome was used as a negative control. P-values were from 2-sample t-test. Null hypothesis: enrichment on GLC3 promoter equals the enrichment on HSP promoters. Protein factors were ranked from highest to lowest by "FC GLC3 over HSP".

The following figure supplements are available for figure 1:

Figure supplement 1. Reporter RNA was enriched on the CoTrIP plasmid.

Figure supplement 2. Rvb1/Rvb2 form cytoplasmic granules that are not co-localized with P-body during glucose starvation.

found Rvb1/Rvb2 are highly enriched on GSY1, GLC3 and HXK1 promoters but not HSP30, HSP26 or HSP104 promoters, which is consistent with our CoTrIP results (Figure 2C). $\mathrm{Rvb1/Rvb2}$ are significantly more enriched on the proximal promoters of the transcriptionally upregulated, poorly translated genes versus the transcriptionally upregulated and well translated genes and the average genome (Figure 2D; Figure 2-supplement figure 4). More generally we found that, for genes that show a greater than 3 -fold increase in mRNA levels during glucose starvation, their promoters are significantly more enriched for Rvb2 binding. Previously we had found that Hsf1-binding sequences were sufficient to exclude mRNAs from mRNP granules during glucose starvation (10). Interestingly we found that glucose starvation induced Hsf1target promoters have no difference in Rvb1/Rvb2 binding than an average gene, and significantly lower Rvb1/Rvb2 enrichment than stress induced non-Hsf1 targets (Figure 2B).

Enrichment peaks of Rvb1/Rvb2 were called using the macs algorithm (32). Consistently, enrichment peaks of
Rvb1/Rvb2 were identified on the promoter regions of the Class II alternative glucose metabolism genes but not the Class I heat shock genes (Figure 2-supplement figure 5). Rvb1 and Rvb2 also show a highly overlapped enrichment pattern across the genome, but neither of them shows overlapped enrichment with the negative control Pgk1 (Figure 2-supplement figure 3). Structural studies have shown that Rvb1/Rvb2 can form a dodecamer complex. Their overlapped enrichment also indicates that Rvb1 and Rvb2 may function together.

\section{Rvb1/Rvb2 are co-transcriptionally loaded on the alternative glucose metabolism mRNAs}

Although Rvb1/Rvb2 are predominantly considered to act on DNA, they are also found to interact with various mRNAs and regulate mRNA translation and stability $(27,33)$. We next sought to test whether Rvb1/Rvb2 established similar enrichment patterns on mRNAs. To test the interaction, we performed RNA ImmunoPrecipitation (RIP) on Rvb1, Rvb2, 
bioRxiv preprint doi: https://doi.org/10.1101/2021.10.17.464753; this version posted October 18, 2021. The copyright holder for this preprint (which was not certified by peer review) is the author/funder, who has granted bioRxiv a license to display the preprint in perpetuity. It is made available under aCC-BY 4.0 International license.

A

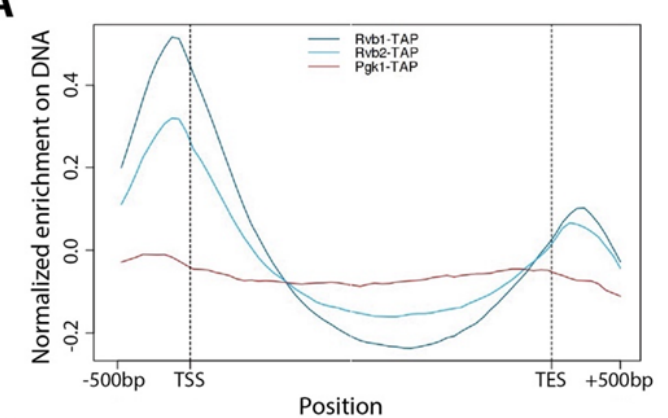

B

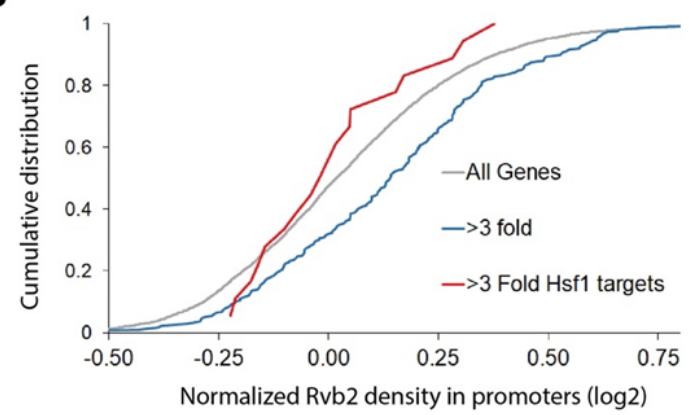

C

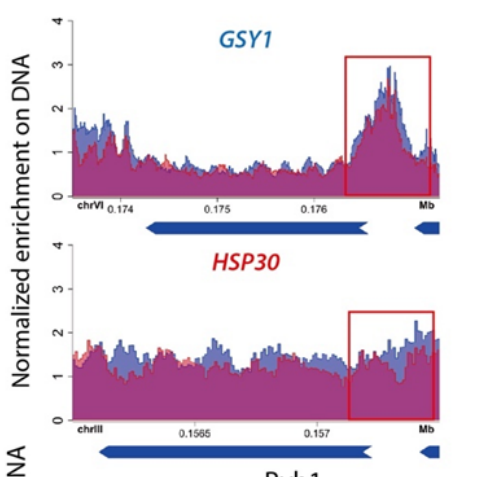

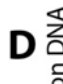

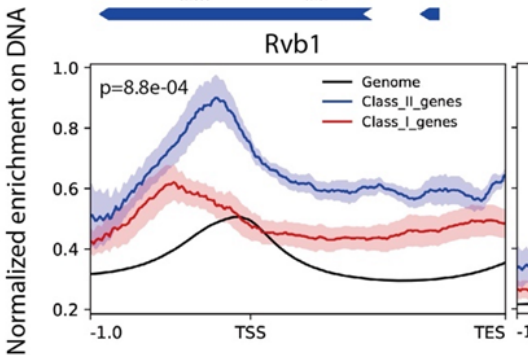

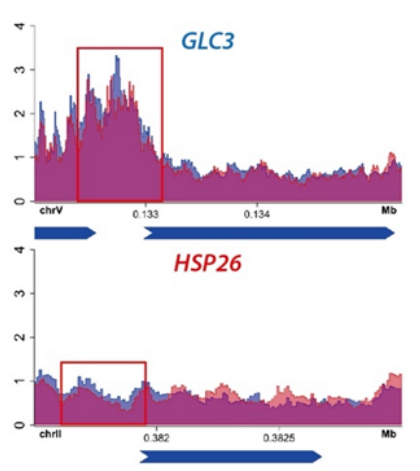

Rvb2

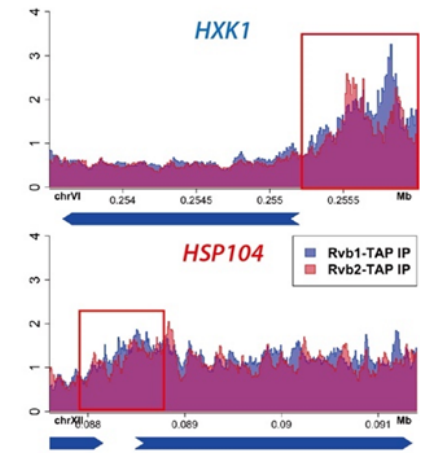

Pgk1

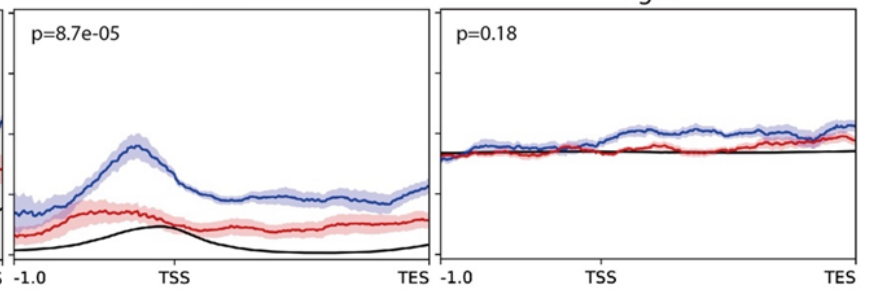

Fig. 2. Rvb1/Rvb2 are enriched at the promoters of endogenous alternative glucose metabolism genes. (A) Rvb1/Rvb2 are enriched on promoters and nascent gene bodies. ChIP-seq of cells in 10-minute glucose starvation. X-axis: normalized scale of all genes containing -500 bp to TSS, TSS to TES and TES to +500 bp. TSS: transcription start site. TES: transcription end site. Y-axis: normalized density of target protein on the loci. Normalized density: RPKM of ChIP over RPKM of input. Input: $1 \%$ of the cell lysate. Rvb1/Rvb2/Pgk1 are C-terminally fused with TAP tag and immunoprecipitated by lgG-conjugating breads. TAP: tandem affinity purification tag. Pgk1: a negative control that considered as non-interactor on the genome. (B) Cumulative distribution of Rvb2's enrichment on genes. X-axis: log2 scale of Rvb2 ChIP read counts over Pgk1 ChIP read counts from -500 bp to TSS. Y-axis: cumulative distribution. $>3$ fold: genes that have more than 3-fold transcriptional induction during 10-minute glucose starvation. >3-fold Hsf1 targets: genes that have more than 3-fold transcriptional induction and are Hsf1-regulated. List of genes are in the supplementary. (C) Representative gene tracks showing Rvb1/Rvb2's enrichment. X-axis: gene track with annotation (in Mb). Arrow's orientation shows gene's orientation. Y-axis: normalized density of Rvb1/Rvb2 over Pgk1. Normalized density: RPKM of ChIP over RPKM of input. Class I genes are labeled in red and Class II genes are labeled in blue. Promoters are highlighted by red rectangles. (D) Enrichment profile of Rvb1/Rvb2 on Class I, II genes and genome. X-axis: normalized scale of genome containing -500 bp to TSS, TSS to TES. TSS: transcription start site. TES: transcription end site. Y-axis: RPKM of ChIP over RPKM of input. P-values are from 2-sample t-test. Null hypothesis: normalized density from -500 bp to TSS on Class II promoters equals on Class I promoters.

The following figure supplements are available for figure 2:

Figure supplement 3. (A) Western validation of ChIP-seq (B) Rvb1 and Rvb2's enrichment regions are highly likely overlapped.

Figure supplement 4. List of Class I upregulated and high-ribo genes and Class II upregulated and low-ribo genes.

Figure supplement 5. List of peaks called of Rvb1/Rvb2 on the genome.

and the negative control wild-type (WT) strain followed by RT-qPCR in both log phase and 15-minute glucose starved cells. Consistently, during 15 minutes of glucose starvation, Rvb1/Rvb2 are significantly more enriched on the mRNAs of the Class II alternative glucose metabolism genes versus the Class I heat shock genes (Figure $3 \mathrm{~A}$ ). Rvb2 is specifically highly enriched on GSY1 mRNA, where it is around 20-fold more enriched than on HSP 30 mRNAs. However, in glucoserich log phase conditions, Rvb1/Rvb2 are generally less enriched on the mRNAs compared to starvation conditions. Additionally, in log phase, Rvb1/Rvb2 do not show differential enrichment between the alternative glucose metabolism genes and the heat shock genes. (Figure 3-supplement figure
$6)$.

Since Rvb1/Rvb2 are enriched on both promoters and mRNAs of Class II alternative glucose metabolism genes, we hypothesized that $\mathrm{Rvb} 1 / \mathrm{Rvb} 2$ are loaded from the interacting promoters to the nascent mRNAs via the transcription process. To test this, we eliminated the effects from the ORF sequences by designing a pair of reporter mRNAs with a uniform $C F P$ ORF but driven by either the GLC3 promoter or HSP26 promoter (Figure 3B). Interestingly, although the mRNA transcribed virtually identical mRNA sequences (10), Rvb1/Rvb2 are significantly more enriched on the mRNA driven by the GLC3 promoter compared to the one driven by the HSP 26 promoter during 15 minutes of glucose star- 


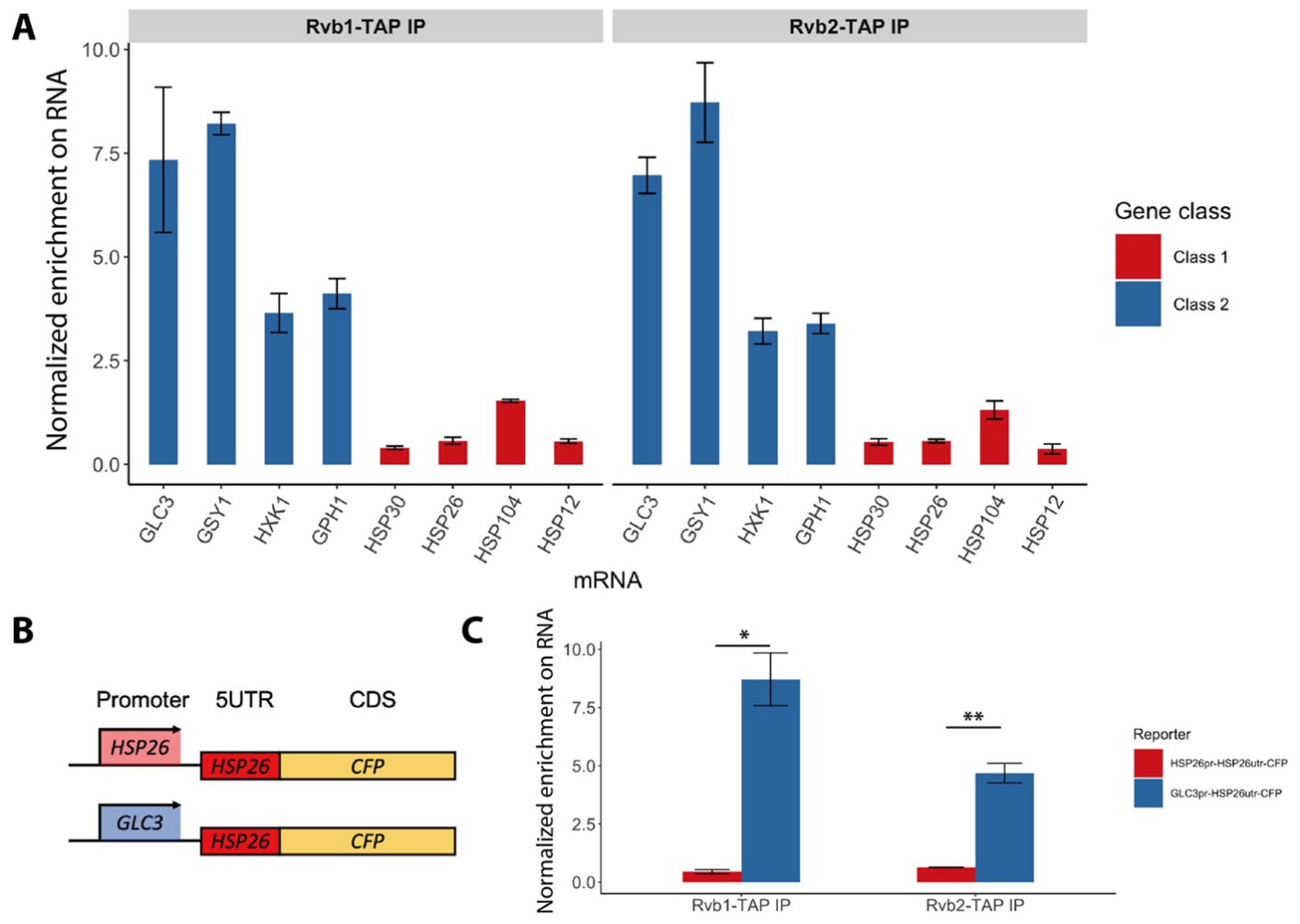

Fig. 3. Rvb1/Rvb2 are co-transcriptionally loaded on the alternative glucose metabolism mRNAs. (A) Rvb1/Rvb2's enrichment on endogenous mRNAs in 15-minute glucose starvation. RNA immunoprecipitation qPCR of cells in 15-minute glucose starvation. Error bars are from 2 biological replicates. X-axis: 4 Class I mRNAs labeled in red and 4 Class II mRNAs in blue. Y-axis: Ct values were firstly normalized by internal control $A C T 1$, then normalized by input control, finally normalized by the wild-type immunoprecipitation control group. Input: $1 \%$ of the cell lysate. (B) A schematic view of the reporter mRNA only swapping the promoter. 5UTR: 5' untranslated region. CDS: coding sequence. CFP: cyan fluorescent protein. (C) Rvb1/Rvb2's enrichment on the reporter CFP mRNAs in 15-minute glucose starvation. RNA immunoprecipitation qPCR of cells in 15-minute glucose starvation. X-axis: HSP26 promoter-driven reporter mRNA labeled in red and GLC3 promoter-driven mRNA in blue. Y-axis: Ct values were firstly normalized by internal control $A C T 1$, then normalized by input control. Input: $1 \%$ of the cell lysate. Standard deviations are from 2 biological replicates. Statistical significance was assessed by 2 -sample t-test $\left({ }^{*} p<0.05,{ }^{* *} p<0.01\right)$. Null hypothesis: the enrichment on the 2 reporter mRNAs is equal.

The following figure supplements are available for figure 3 :

Figure supplement 6. Rvb1/Rvb2 did not show differential enrichment between Class I and Class II mRNAs in glucose-rich log-phase cells.

vation (Figure 3C). This suggests that only the promoter itself can determine the transcribed mRNA's interaction with

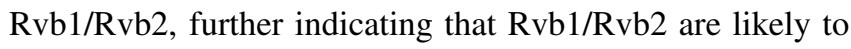
be co-transcriptionally loaded from the promoters to nascent mRNAs.

\section{Engineered Rvb1/Rvb2 tethering to mRNAs directs the cyto- plasmic localization and repressed translation}

As Rvb1/Rvb2 were found to be located at both promoters in the nucleus and associated with mRNAs in the cytoplasm, we asked whether Rvb1/Rvb2 have an impact on the cytoplasmic fates of bound mRNAs. To test this, we engineered interactions between Rvb1 or Rvb2 and the mRNAs transcribed from various promoters of Class I heat shock genes (e.g., HSP 30, HSP26). We took advantage of the specific interaction between a phage-origin PP7 loop RNA sequence and the PP7 coat protein (34). Here, in our engineered strains, a reporter construct consists of a promoter of interest, a nanoluciferase reporter ORF for measuring protein synthesis, a PP7 loop to drive the engineered interaction, and an MS2 loop for the mRNA subcellular visualization. Along with the reporter, Rvb1 or Rvb2 are fused with PP7-coat protein to establish binding on the reporter mRNA (Figure 4A). As previously shown Rvb1/Rvb2 do not display strong binding on the promoters and mRNAs of Class I heat shock genes (Figure 2C, 3A) (e.g., HSP30). Therefore, we specifically engineered the interaction between Rvb1 or Rvb2 and two types of mRNAs driven by the Class I heat shock promoters (HSP30/HSP26). Strikingly, binding of both Rvb1 and Rvb2 alters the cytoplasmic fates of these Class I heat shock mRNAs to be similar to the Class II alternative glucose metabolism mRNAs (Figure 4; Figure 4-figure supplement 7, 8). Taking HSP3O promoter-driven reporter mRNA as an example, during glucose starvation the binding of Rvb1 and Rvb2 reduces protein synthesis by $\sim 35 \%$ and $\sim 50 \%$ respectively (Figure $4 \mathrm{~B}$ ). It is important to consider that final protein abundance is determined by both mRNA levels and translation. Interestingly, we observed an increase in mRNA abundance when Rvb1 or $\mathrm{Rvb} 2$ are tethered to the reporter mRNA (Figure 4C). When the translational efficiency was normalized by the mRNA abundance, we were surprised to observe binding of either Rvb1 or Rvb2 reduces translational efficiency by greater than $\sim 70 \%$ during glucose starvation (Figure 4D). Additionally, $\mathrm{Rvb1/Rvb2}$ binding does not significantly repress the translational efficiency of mRNA in glucose-rich unstressed cells, indicating that Rvb1/Rvb2 has a more significant effect on 
bioRxiv preprint doi: https://doi.org/10.1101/2021.10.17.464753; this version posted October 18, 2021. The copyright holder for this preprint (which was not certified by peer review) is the author/funder, who has granted bioRxiv a license to display the preprint in perpetuity. It is made available under aCC-BY 4.0 International license.

A

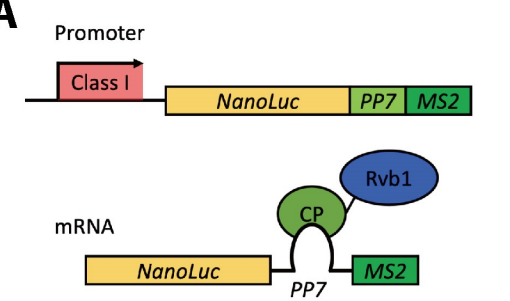

C
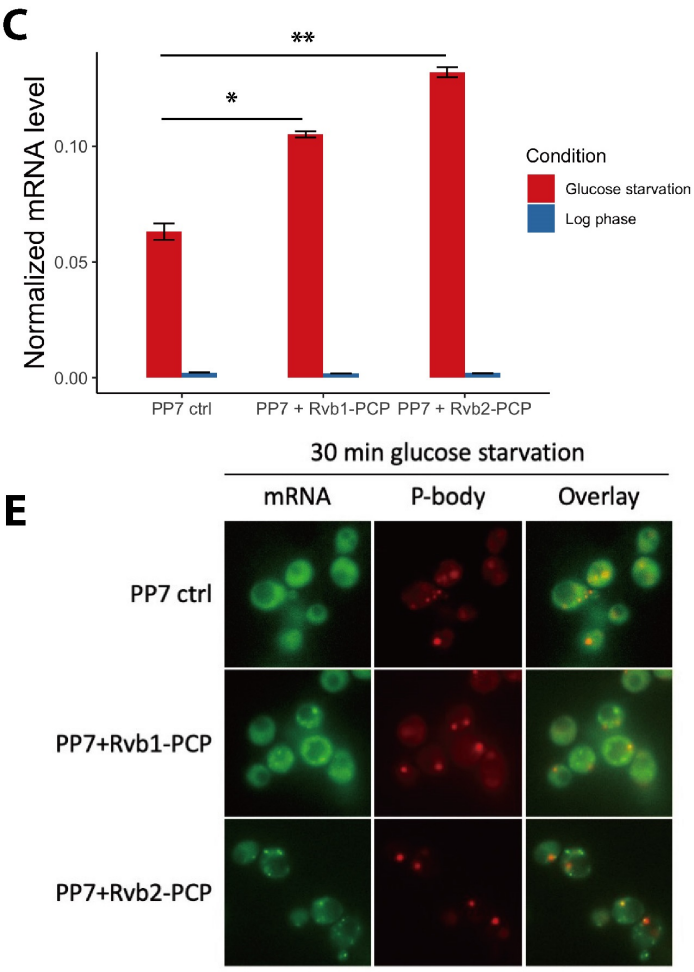
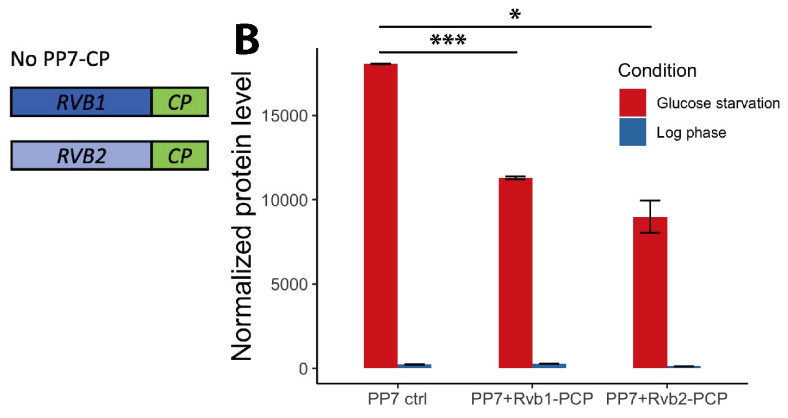

D
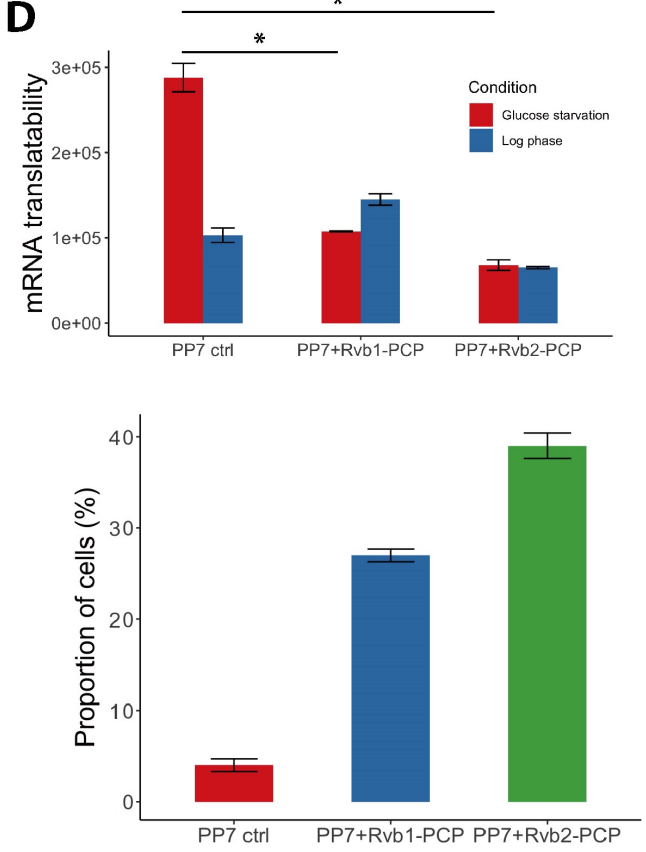

Fig. 4. Engineered Rvb1/Rvb2 tethering to mRNAs directs cytoplasmic granular localization and repressed translation. (A) A schematic view of Rvb-tethering methodology. The reporter mRNA contains promoter of interest (Class I promoters), nanoluciferase CDS, PP7 loop sequence, and $12 X M S 2$ sequence. Rvb1 or Rvb2 are C-terminally fused with PP7-coat protein (CP). Upper panel shows cloning strategy and lower panel shows mRNA's situation upon engineering. (B) protein synthesis of HSP30 promoter-driven reporter mRNA in log phase and 25-minute glucose starvation. Y-axis: nanoluciferase synthesized within 5-minute time frame. Nanoluciferase reading was subtracted by the nanoluciferase reading of cycloheximide added 5 minutes earlier. (C) mRNA levels of HSP30 promoter-driven reporter mRNA in log phase and 15-minute glucose starvation. Y-axis: Ct values of reporter mRNAs were normalized by the internal control ACT1. (D) Translatability of HSP30 promoter-driven reporter mRNA in log phase and 15-minute glucose starvation. mRNA translatability: normalized protein level over normalized mRNA level. (B, C, D) Log phase is labeled in blue and glucose starvation in red. Error bars are from 2 biological replicates. Statistical significance was assessed by 2-sample t-test. Null hypothesis: experimental groups and control groups have equivalent results $\left({ }^{*} \mathrm{p}<0.05,{ }^{* *} \mathrm{p}<0.01,{ }^{* * *} \mathrm{p}<0.001\right)$. PP7 ctrl: negative control, cells only have the reporter mRNA with PP7 loop. PP7+Rvb1-PCP: Rvb1 is tethered to mRNA. PP7+Rvb2-PCP: Rvb2 is tethered to mRNA. (E) Live imaging showing the subcellular localization of the HSP30 promoter-driven reporter mRNA in 30-minute glucose starvation. Reporter mRNA is labeled by the MS2 imaging system. P-body is labeled by marker protein Dcp2. PP7 ctrl: negative control, cells only have the reporter mRNA with PP7 loop. PP7+Rvb1-PCP: Rvb1 is tethered to mRNA. PP7+Rvb2-CP: Rvb2 is tethered to mRNA. Right: quantification of the subcellular localization of the reporter mRNA.Y-axis: percentage of cells that have the reporter mRNA-containing granule foci. $\mathrm{N}=200$. Error bars are from 2 biological replicates.

The following figure supplements are available for figure 4

Figure supplement 7. Engineered Rvb1/Rvb2 tethering to HSP30 promoter-driven reporter mRNA directs cytoplasmic granular localization and repressed translation. Figure supplement 8. Engineered Rvb1/Rvb2 tethering to HSP26 promoter-driven reporter mRNA directs cytoplasmic granular localization and repressed translation. Figure supplement 9. Ribosome occupancy of endogenous alternative glucose metabolism mRNAs was quickly induced after glucose replenishment.

mRNAs when mRNP granules have visibly formed (Figure 4-figure supplement 7, 8).

Since the translation of the mRNAs bound by Rvb1/Rvb2 was reduced, we further visualized the subcellular localization of those mRNAs. Consistent with reduced translation, Rvb1/Rvb2-tethering significantly increases the granular localization of the heat shock mRNA reporters (Figure 4E; Figure 4-figure supplement 7, 8). Taking HSP3O promoter-driven reporter mRNA as an example, only $4 \%$ of the cells form HSP30 promoter-driven mRNA-containing granules when the mRNA is not bound by Rvb1 or Rvb2, yet Rvb1-tethering increases the mRNA's granular localization to $27 \%$ of the cells and Rvb2-tethering increases the mRNA's granular localization to $39 \%$ of the cells (Figure 4E). Furthermore, the binding of Rvb1 and Rvb2 to mRNA increases the formation of granules that are non-colocalized with a P-body marker (Figure 4-figure supplement 7). This indicates that Rvb2 guides the interacting mRNA to the presumed stress granule. To further eliminate any potential artifacts caused by the C-terminal modification on Rvb1/Rvb2, the negative 
bioRxiv preprint doi: https://doi.org/10.1101/2021.10.17.464753; this version posted October 18, 2021. The copyright holder for this preprint (which was not certified by peer review) is the author/funder, who has granted bioRxiv a license to display the preprint in perpetuity. It is made available under aCC-BY 4.0 International license.
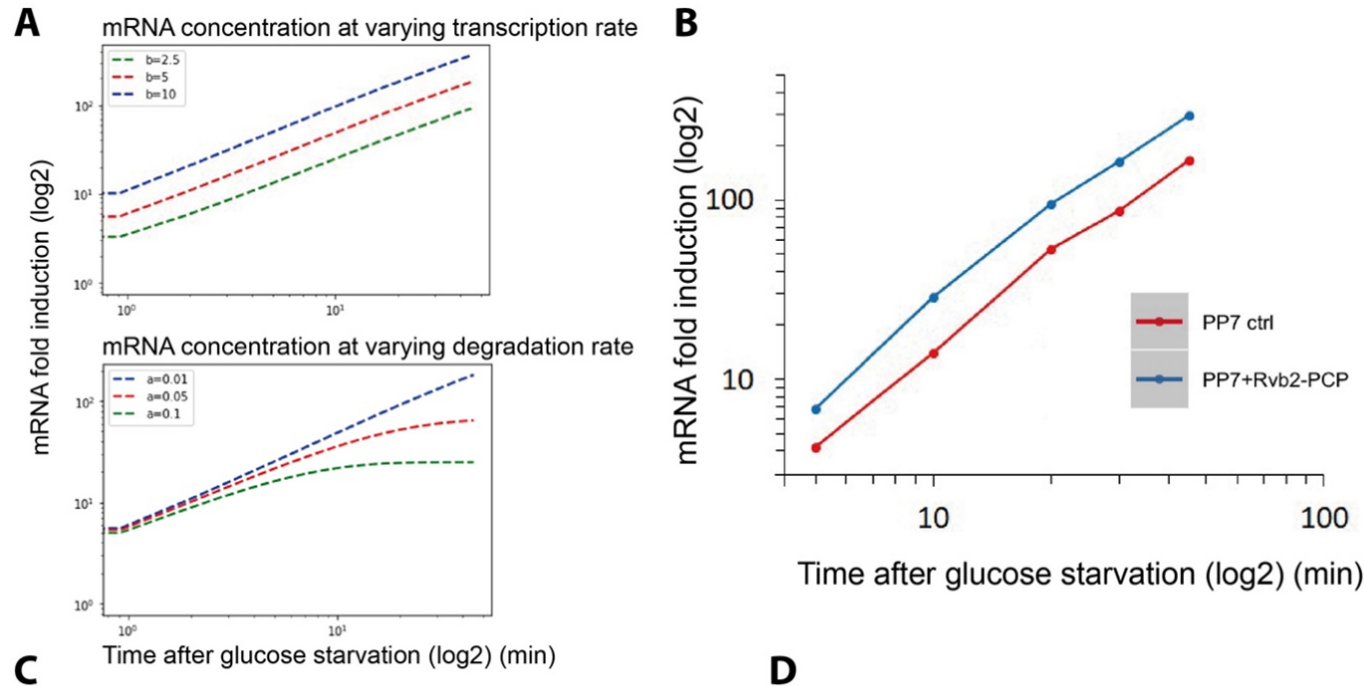

C
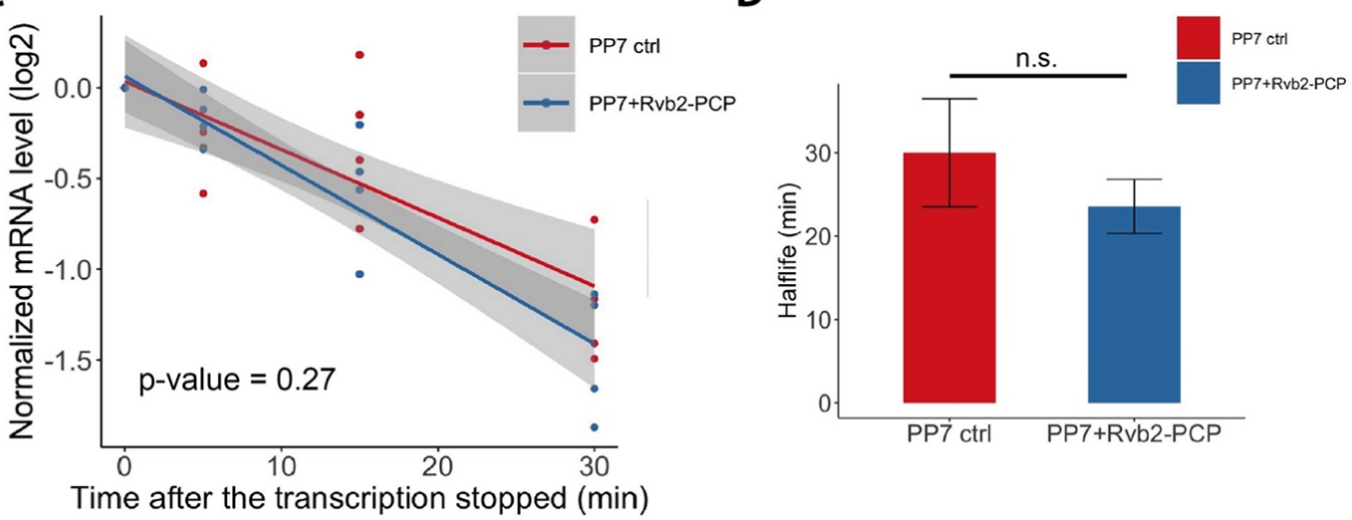

Fig. 5. Engineered Rvb1/Rvb2 binding to mRNAs increases the transcription of corresponding genes. (A) Mathematical modeling on mRNA abundance upon varied transcription rates and varied mRNA degradation rates. $\mathrm{X}$-axis: time (minute) after glucose is removed. Y-axis: mRNA fold induction compared to pre-stress condition. Modeling function: $\Delta X(t)=\left(\frac{\beta}{\alpha}-X_{0}\right)\left(1-e^{-\alpha t}\right), \frac{d X}{d t}=\beta-\alpha X$. $\alpha /$ a denotes the degradation rate constant. mRNA is produced at a constant rate $(\beta / \mathrm{b})$. mRNA concentration is $(X)$. (B) mRNA fold induction of Rvb2-tethered mRNAs and non-tethered mRNAs over time. Reporter mRNA is HSP30 promoter driven. X-axis: time (minute) after glucose is removed. Y-axis: mRNA fold induction compared to pre-stress condition (log scale). PP7 ctrl: negative control, cells only have the reporter mRNA with PP7 loop, labeled in blue. PP7 + Rvb2-PCP: Rvb2 is tethered to mRNA, labeled in red. (C) mRNA decay curve of HSP30 promoter-driven reporter mRNAs. X-axis: after cells were starved for 15 minutes, time (minute) after stopping the transcription using 1,10-phenanthroline. Y-axis: log2 scale of normalized mRNA levels. Ct values of reporter mRNAs were normalized by the internal control ACT1. Statistical significance was achieved by linear regression modeling. Null hypothesis: the mRNA levels of experimental and control groups are equivalent. PP7 ctrl: negative control, cells only have the reporter mRNA with PP7 loop, labeled in blue. PP7 + Rvb2-PCP: Rvb2 is tethered to mRNA, labeled in red. (D) Half-lives of HSP30 promoter-driven reporter mRNAs. Error bars are from 4 biological replicates. Statistical significance was achieved by 2-sample t-test. Null hypothesis: the mRNA half-lives of experimental and control groups are equivalent.

controls were tested where Rvb1 or Rvb2 are fused with PP7 coat protein, but the mRNA does not have the PP7 loop. The negative control strains did not show a decrease in translatability of the reporter mRNAs in glucose starvation. It indicates translation only decreases and the mRNA granular localization level only increases when the full Rvb-mRNA interaction was established (Figure 4-figure supplement 7, 8). These results support the ability of Rvb1/Rvb2 to suppress the translation of the binding mRNAs, potentially through sequestering the mRNAs into cytoplasmic granules.

The coupling of induced transcription and repressed translation of the Class II alternative glucose metabolism genes may be an important adaptation for cells to survive from stress conditions. Results showed that after replenishing the glucose to the starved cells, the translation of those genes is quickly induced, with an $\sim 8$-fold increase in ribosome occupancy 5 minutes after glucose readdition for Class II mRNAs (Figure 4-figure supplement 9). This indicates the po- tential biological role of the stress granule as a repository for these translationally repressed Class II mRNAs during stress that does not preclude these mRNAs from potentially being quickly released and translated once the stress is removed.

\section{Engineered Rvb1/Rvb2 binding to mRNAs increases the transcription of corresponding genes}

Interestingly, Rvb1/Rvb2 not only suppress the translation of bound mRNAs, but also increases the abundance of the interacting mRNAs by more than 2-fold (Figure 4C). There are two possibilities for this increased mRNA abundance by Rvb1/Rvb2-tethering: increased transcription and/or slower mRNA decay. To address this, we performed time-course measurements on the HSP30 promoter-driven reporter mRNA abundance in $0,5,10,15,30,45$ minutes of glucose starvation. Here, we compared the mRNA abundance when mRNA is bound by Rvb2 and when mRNA is not bound by Rvb2 as a control. A mathematical modelling ap- 
proach was performed to predict the mRNA induction abundance change caused by varied transcriptional efficiency or varied decay rate (Figure 5A function) (35). In the model, we assumed that mRNA level is mainly dependent on the transcriptional and decay rates, and these parameters stay constant over the course of induced expression during glucose starvation. From the mathematical modelling, mRNA fold induction differs between varied transcriptional induction versus mRNA decay changes. If transcriptional rates vary, differences in mRNA levels are the same (equal distance shift) at each time point on a log-log scale (Figure 5A upper panel). While if mRNA decay varies, although little difference is seen in mRNA abundance in early glucose starvation, our simulation predicts increasing differences in mRNA abundance at later time points of glucose starvation. (Figure $5 \mathrm{~A}$ lower panel). By comparing experimental measurements of mRNA induction of the Rvb2-tethered condition to the unbound control condition, the mRNA induction differences are similar at each time point. When Rvb2 binds to the mRNA, the abundance of the mRNA is constantly greater than the unbound mRNA at all time points during glucose starvation, indicating that the greater mRNA abundance is mainly due to greater transcription differences driven by the Rvb2 binding (Figure 5B). Also, the mRNA abundance does not differ in log phase, which indicates the effect is potentially stressinduced only.

To further experimentally validate that the greater mRNA abundance caused by Rvb2 binding is due to increased transcription and not slower decay, we stopped cellular transcription after 15 minutes of glucose starvation by treatment with the transcription inhibitor drug 1,10 phenanthroline. Then we performed time-course measurements on mRNA abundance and compared the decay of mRNAs with and without Rvb2 binding. Consistently, mRNAs bound by Rvb2 decay at a slightly but not significantly faster rate, not a slower rate (Figure 5C). Whether or not bound by $\mathrm{Rvb2}$, the reporter mRNA has around a 25-minute half-life (Figure 5D). These results further point to Rvb2 mRNA tethering driving transcriptional upregulation. Since Rvb2 is targeted to the mRNA, it is likely that local recruitment of Rvb2 to the nascently transcribed mRNA increases the local concentration of Rvb2 protein to the vicinity of the regulatory region of the corresponding gene, further showing the connections between the transcriptional and translational processes.

\section{Discussion}

In fluctuating environments, cells must quickly adjust the expression of different genes dependent upon cellular needs. Here, our results demonstrate a novel function of the AAA+ ATPases Rvb1/Rvb2 in the cytoplasm, and a novel mechanism of Rvb1/Rvb2 in coupling the transcription, mRNA cytoplasmic localization, and translation of specific genes (Figure 6). We identified Rvb1/Rvb2 as enriched protein factors on the promoters of the Class II alternative glucose metabolism genes that are upregulated in transcription but downregulated in translation during glucose starvation. Results showed that Rvb1/Rvb2 have a strong pre- ferred interaction with both promoters and mRNAs of these genes, suggesting Rvb1/Rvb2 are loaded from enriched promoters to the nascent mRNAs. More interestingly, when we tethered Rvb1/Rvb2 to the mRNAs, the binding of Rvb1/Rvb2 had a strong impact on reducing mRNA translation and increasing the mRNA granular localization, suggesting that co-transcriptional loading of Rvb1/Rvb2 directs post-transcriptional mRNA fate in the cytoplasm. Additionally, Rvb1/Rvb2's interaction with the mRNA can also induce transcription of the corresponding genes, further indicating that Rvb1/Rvb2 couple the transcription and translation of the interacting genes.

It is not clear how tethering Rvb1/Rvb2 to an mRNA reporter increases transcription of the corresponding DNA locus. Rvb1/Rvb2 were initially found to be associated with many chromatin-remodeling and transcription related complexes. This has further been expanded on and several studies have demonstrated a chaperone-like activity in the formation of various complexes including the assembly of chromatin remodeling complexes and RNA polymerase II (2022). It may be that recruiting Rvb1/Rvb2 to the nascent RNA increases the local concentration, driving further enhancement of transcription-related processes. It is also intriguing to think about Rvb1/Rvb2's reporter role in escorting client proteins to large macromolecular complexes. Like their escorting protein function, it is plausible to hypothesize that they might have additional functions in escorting mRNAs to the large macromolecule stress granule complex in stressful conditions.

The coupling of transcription and translation of specific genes may be an important adaptation for cells to survive during stress conditions. It has been postulated that to save energy during stress, mRNAs are temporarily stored in the cytoplasmic granules associated with inactive translation instead of mRNA decay $(9,11,36-38)$. Our results show that, after replenishing glucose to the starved cells, the translation of those genes is quickly induced (Figure 4-figure supplement 9). The stress-induced phase-separated granules may serve as temporary repositories for the inactive translating mRNAs of many genes that are regulated by Rvb1/Rvb2 in glucose starvation and involved in alternative glucose metabolism pathways. From the perspective of cell needs, many alternative glucose metabolism genes are involved in glycogen synthesis, which may be superfluous for survival during times of complete glucose starvation, but the cell may want to produce them as quickly as possible upon glucose replenishment to drive quick protein synthesis. The special coupling of increased transcription but repressed translation mediated by $\mathrm{Rvb} 1 / \mathrm{Rvb} 2$ may serve as an emergency but prospective mechanism for cells to precisely repress the translation of these alternative glucose metabolism mRNAs during stress but be able to quickly translate these pre-stored mRNAs once the cells are no longer starved (39). Also, the genes regulated by Rvb1/Rvb2 may be dependent on the type of stresses. We observed this mechanism of gene expression control on alternative glucose metabolism genes during glucose starvation stress. It will be interesting to test if there is similar regula- 
Nucleus

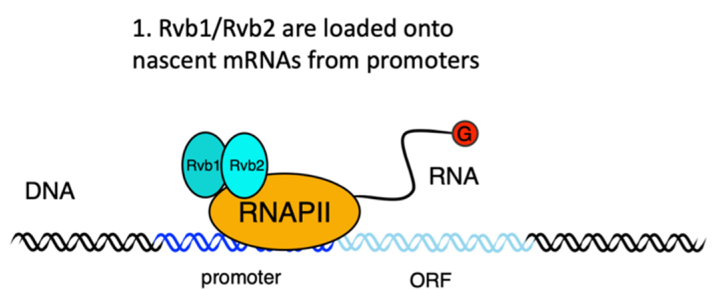

3. Rvb1/Rvb2 tethering on mRNAs induces the transcription on DNA
Cytoplasm 2. Rvb1/Rvb2 directs binding mRNAs to granules and inactive translation

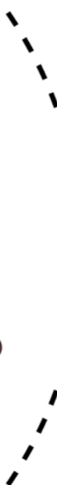

Fig. 6. A working illustration of Rvb1/Rvb2's mechanism in coupling the transcription and translation of interacting genes. First, Rvb1/Rvb2 are recruited by specific promoters and loaded onto the nascent mRNAs during glucose starvation. Then Rvb1/Rvb2 escort the interacting mRNAs to the cytoplasm and direct their localization to cytoplasmic granules and associated repressed translation. Also, forced Rvb binding on an mRNA drives an increase in the transcription of the corresponding genes, further showing the coupling of transcription and translation.

tion on different sets of genes that are related to other types of stresses, such as heat shock and osmotic stress responses.

To further understand the function of Rvb1/Rvb2 mechanistically, it is crucial to understand how Rvb1/Rvb2 are recruited to these specific promoters. We prefer the hypothesis that the recruitment of Rvb1/Rvb2 is mediated by other DNA-binding proteins, as we were unable to identify specific binding motifs of Rvb1/Rvb2 from our ChIP-seq data. As we found that Rvb1/Rvb2 are generally enriched on the promoters of transcriptionally upregulated mRNAs we favor a model in which the default is for Rvb1/Rvb2 to be recruited to active transcription sites. This fits with previous data that Rvb1/Rvb2 are required to maintain expression of many inducible promoters including galactose-inducible transcripts (40). While Rvb1/Rvb2 are generally recruited to the promoters of induced mRNAs during glucose starvation, we find that Hsf1-regulated promoters circumvent this recruitment through an unknown mechanism, as the transcriptionally upregulated Hsf1 targets show reduced recruitment relative to non-Hsf1 targets (Fig 2B). Intriguingly, Hsf1-regulated genomic regions have been found to coalesce during stressful conditions $(41,42)$. It will be interesting to explore whether Rvb1/Rvb2 may be excluded from these coalesced regions in future studies.

This study provides new insights into how gene expression is controlled under stressful conditions, including how mRNAs can be targeted to stress induced mRNP granules. It also identifies Rvb1/Rvb2 as key proteins connecting discrete steps of gene expression across cellular compartments. In mammalian cells overexpression of the $R V B 1 / 2$ homologs, $R U V B L 1 / 2$, are correlated with tumor growth and poor prognosis in several cancer types, yet precise mechanisms for how these proteins impact cancer progression is unclear (43-45). It is important to further study the role these proteins have on connecting gene expression in different conditions to better understand how they may be impacting cancer progression in mammalian cells.

\section{Materials and methods}

\section{Yeast strains and plasmids}

The yeast strains and plasmids used in this study are listed in Supplementary file 1 and the oligonucleotides used for the plasmid construction, yeast cloning and RT-qPCR are described in Supplementary file 2. The strains were created through genomic integration of a linear PCR product, or a plasmid linearized through restriction digest or the transformation of an episomal vector. The background strain used was W303 (EY0690), one laboratory strain that is closely related to $\mathrm{S} 288 \mathrm{C}$. In yeast cloning for the C-terminal fusion on the endogenous proteins (e.g. Rvb1-mNeongreen, Rvb1PP7CP, Rvb1-TAP etc.), we used plasmids of Pringle pFA6a and pKT system $(10,46,47)$, gifts from the E. K. O'Shea laboratory and the K. Thorn laboratory. We modified the pFA6a and pKT plasmids by inserting in the peptides of interest into the plasmids. The primers used to amplify the fragments from these plasmids contain 2 parts from 3' to 5': a uniform homolog sequence to amplify the plasmid and a homolog sequence to direct inserting the fragments to the genomic loci of interest. The fragments were transformed into the yeasts and integrated to the genome by homologous recombination. The integrations were confirmed by genomic DNA PCR (Yeast DNA Extraction Kit from Thermo Fisher). In the cloning of the reporter strains, we used a strain that was derived from W303 and has one-copied genomic insertion of MYOpr-MS2CP-2XGFP and an endogenous fusion Dcp2-mRFP (10), as the background strain. Further we transformed the linearized MS2-loop-containing reporter plasmids into the strain by restriction digest and genomic integration. RT-qPCR was performed to verify the one-copied genomic integration. To generate the MS2-loop-containing reporter plasmids (e.g., ZP207 pRS305-HSP30prUTR-nLucPEST-12XMS2-tADH1), we started from the plasmid ZP15 pRS305-12XMS2-tAdh1 (10). ZP15 was linearized by the restriction enzymes SacII and NotI (NEB). Promoter fragments, nanoluciferase-pest CDS fragments were inserted into linearized ZP15 using Gibson Assembly. Promoter se- 
quences were amplified by PCR from the W303 genomic DNA. Nanoluciferase-pest CDS was amplified by PCR from the geneblock (48). To generate the PP7-MS2-containing reporter plasmids (e.g., ZP296 pRS305-HSP30prUTR-nLucPEST-1XPP7-12XMS2-tADH1), ZO680 and ZO679 were firstly annealed using the primer annealing protocol described by Thermo Fisher. ZP15 was linearized by restriction enzymes BamHI and NotI. Then annealed oligos were inserted into linearized ZP15 by T4 ligation to generate ZP440. ZP440 was further linearized by restriction enzymes SacII and NotI. Promoter fragments, nanoluciferasepest CDS fragments were inserted into linearized ZP440 using Gibson Assembly. In the cloning of the CoTrIP experiments, detailed procedures were described in the "CoTrIP and CoTrIP analysis".

\section{Yeast growth and media}

The background yeast strain w303 (EY0690) was used for all experiments. For cells cultured in the functional experiments, cells were streaked out on the yeast extract peptone dextrose (YPD) agarose plate (BD) from the frozen stocks and grew at $30{ }^{\circ} \mathrm{C}$ for 2 days. Single colony was selected to start the over-night culture for each biological replicate. Cells were grown at $30^{\circ} \mathrm{C}$ in batch culture with shaking at 200 r.p.m. in synthetic complete glucose medium (SCD medium: yeast nitrogen base from RPI, glucose from Sigma-Aldrich, SC from Sunrise Science). When the OD660 of cells reached 0.4, half of the culture was harvested as the pre-starved sample. The other half of the culture was transferred to the prewarmed synthetic complete medium lacking glucose (SC -G medium) by centrifugation method. Cells are centrifuged at $3000 \mathrm{xg}$, washed once by SC medium and resuspended in the same volume as the pre-starvation medium of SC medium. Glucose starvation was performed in the same 200 r.p.m shaking speed and $30^{\circ} \mathrm{C}$. The length of the glucose starvation time varies from 10 minutes to 30 minutes depending on the experiments.

\section{CoTrIP and CoTrIP analysis}

The protocol was developed based on (29). ZP64 PRS406CMV-lacI-FLAG was integrated into the W303 yeast background by linearization withing the URA3 gene with BstBI digestion and transformation into yeast. The CoTrIP plasmid was constructed by modifying the ZP66 pUC-TALO8 plasmid which contains 8 copies of the Lac operator. The EcoRI sites in ZP66 were mutated to NotI using QuikChange II Sitedirected mutagenesis kit (Stratagene). Promoter specific reporters driving CFP were inserted into pUC-TALO8-NotI by digesting with NheI followed by Gibson assembly. The plasmid backbone was then digested with NotI, gel purified, ligated, and transformed into yeast using standard Lithium Acetate transformation. $1 \mathrm{~L}$ of yeast were grown overnight in SCD medium -Trp to maintain selection on the CoTrIP plasmid, until an OD660 0.3-0.4. Cells were filtered, washed with SC -G -Trp media, and resuspended in 1L of prewarmed media and grown at $30^{\circ} \mathrm{C}$ for 5 mins. Cells were then refiltered, resuspended in $4 \mathrm{mLs}$ of PBS in a glass petri dish and crosslinked using UV from a Stratalinker $1800(254 \mathrm{~nm}, 9999$ microjoules $\times 100,5 \mathrm{~cm}$ from the UV bulb). Crosslinked cells were pelleted and resuspended in $2 \mathrm{mLs}$ of Buffer $\mathrm{H} 150$ (25mM HEPES KOH pH 7.6, 2mM MgCl2, 0.5mM EGTA, $0.1 \mathrm{mM}$ EDTA, $10 \%$ Glycerol, $150 \mathrm{mM} \mathrm{KCl}, 0.02 \%$ NP40) plus protease inhibitor (P8215 Millipore Sigma) and then dripped into liquid $\mathrm{N} 2$ to be cryogenically ball milled using a Retsch PM100. Ground lysate was clarified by spinning at $3500 \mathrm{xg}$ for $5 \mathrm{mins}$ at $4^{\circ} \mathrm{C}$, isolating the supernatant and spinning at $12 \mathrm{~K}$ for $5 \mathrm{~min}$ at $4^{\circ} \mathrm{C}$. Supernatant was aliquoted and frozen at $-80^{\circ} \mathrm{C} .10 \mu \mathrm{L}$ of unpacked Anti-FLAG® M2 Magnetic Beads (M8823 Millipore Sigma) per sample were pre-washed with Buffer $\mathrm{H}$ 150. $300 \mu \mathrm{L}$ of extract was added to magnetic beads and incubated at $4{ }^{\circ} \mathrm{C}$ for $3 \mathrm{hrs}$ with rotation. Beads were then washed 3 times with Buffer $\mathrm{H} 150$, 3 times with Buffer $\mathrm{H} 300(300 \mathrm{mM} \mathrm{KCl})$, and once with Buffer H 150. $500 \mu \mathrm{g} / \mathrm{mL}$ 3xFLAG peptide (F4799 Millipore Sigma) was diluted in Buffer H 150 and CoTrIP plasmids were eluted with $100 \mu \mathrm{L}$ elution buffer with FLAG peptide. Elutions were taken forward for DNA, RNA, and mass spectrometry. Mass spectrometry was performed through the Yeast Resource Center by James Moresco of the Yates lab and was funded through a P41GM103533 Biomedical Technology Resource Center grant. Data-dependent acquisition of MS/MS spectra was performed with an LTQ-Orbitrap. Tandem mass spectra were extracted from raw files using RawExtract 1.9.9 (49) and were searched against a yeast protein database (http://www.yeastgenome.org) using ProLuCID (50, 51). Enrichment analysis was performed using the CRAPome online data analysis software (https://reprintapms.org/) (30).

\section{ChIP-sequencing}

The protocol was developed based on (52). $100 \mathrm{~mL}$ of yeast were grown overnight in SCD medium, until an OD660 around 0.4. $50 \mathrm{~mL}$ of cells were filtered, washed with $\mathrm{SC}$ $\mathrm{G}$ media, and resuspended in $50 \mathrm{~mL}$ of prewarmed media and grown at $30^{\circ} \mathrm{C}$ for 10 mins. $50 \mathrm{~mL}$ of pre-starved and 50 $\mathrm{mL}$ of 10-minute glucose-starved cell culture was fixed by incubating in the freshly made crosslink buffer (1\% formaldehyde, $10 \mathrm{mM} \mathrm{NaCl}, 0.1 \mathrm{mM}$ EDTA, $5 \mathrm{mM}$ HEPES $\mathrm{pH}$ 7.5), respectively, with gentle shaking at room temperature for 15 minutes. Crosslink was quenched by introducing $0.5 \mathrm{M}$ of glycine for 5 minutes at room temperature. Cells were harvested by centrifugation at $3000 \mathrm{xg}$ at $4{ }^{\circ} \mathrm{C}$, washed twice in the ice-cold TBS buffer (20 mM Tris pH 7.5, $150 \mathrm{mM}$ $\mathrm{NaCl}$ ). Cells were resuspended in $400 \mu \mathrm{L}$ of ChIP lysis buffer (50 mM Hepes-KOH pH 7.5, $150 \mathrm{mM} \mathrm{NaCl}, 1 \mathrm{mM}$ EDTA, $1 \%$ Triton X-100, 0.1\% sodium deoxycholate, $1 \mathrm{mM} \mathrm{PMSF}$, $0.5 \%$ SDS), and lysed by bead-beating (Biospec Products) for 1 minute for 5 times. Lysis was verified under microscope. Lysates were sonicated by Covaris Sonicator to $\sim 500$ bp fragments (130 $\mu \mathrm{L} /$ tube, 105 PIP, 5\% Duty F, 200 cycles/burst, 80 seconds). Lysates were centrifuged at 15,000 $\mathrm{xg}$ at $4^{\circ} \mathrm{C}$ to remove the cell debris and diluted to $1 \mathrm{~mL}$. Save $10 \%$ of the clear lysate to verify the sonication by protein digestion using Pronase, reverse crosslinking, RNA digestion 
using RNase A and running the samples on $1 \%$ agarose gel. $50 \mu \mathrm{L}$ of IgG-Dynabeads per sample was used. The protocol of making the IgG-Dynabeads from Dynabeads M-270 Epoxy (Thermo Fisher) was taken from (53). IgG-Dynabeads were pre-washed 3 times with ChIP lysis buffer. $1 \%$ of the lysate was saved as the input and for Western blotting, respectively. The immunoprecipitation (IP) sample was incubated with IgG-Dynabeads, rotating at $4^{\circ} \mathrm{C}$ for 4 hours. The IP samples were further washed twice by ChIP lysis buffer with $0.1 \%$ SDS, twice by ChIP lysis buffer with $0.1 \%$ SDS and $0.5 \mathrm{M} \mathrm{NaCl}$, once by ChIP wash buffer $(50 \mathrm{mM}$ Tris pH 7.5, $0.25 \mathrm{mM} \mathrm{LiCl,} 1 \mathrm{mM}$ EDTA, 0.5\% NP-40, 0.5\% sodium deoxycholate), and once by TE buffer $\mathrm{pH} 7.5$. Before the last wash, save $10 \%$ of the sample for Western blotting. Western blotting was performed to verify the successful enrichment and purification of the protein of interest. Samples were later eluted from beads in $250 \mu \mathrm{L} 2 \mathrm{X}$ Pronase buffer (50 mM Tris $\mathrm{pH} 7.5,10 \mathrm{mM}$ EDTA, $1 \%$ SDS) at $65^{\circ} \mathrm{C}$ for 10 minutes and beads were removed. Samples were then digested by $1.6 \mathrm{mg} / \mathrm{mL}$ Pronase $(20 \mu \mathrm{L}$ of $20 \mathrm{mg} / \mathrm{mL}$ Pronase, Sigma-Aldrich) at $42^{\circ} \mathrm{C}$ for 2 hours and reverse-crosslinked at $65^{\circ} \mathrm{C}$ for 6 hours. DNA was extracted from the sample using phenol-chloroform, washed once with chloroform, and pelleted using ethanol, washed twice with $75 \%$ ethanol (Sigma-Aldrich), and resuspended in $200 \mu \mathrm{L}$ TE buffer. DNA sequencing libraries were prepared and sequenced following the NextSeq 500 SR 75 protocol at The UCSD IGM Genomics Center (Illumina).

\section{ChIP-sequencing analysis}

Sequencing reads were demultiplexed and the quality was verified by FastQC (54). The reads were mapped to S288C S. cerevisiae genome using Bowtie2 (55). The mapped reads were then sorted, indexed and converted into BAM files using SAMtools (56). Duplicated reads were removed using Picard Tools (57). The genome browser files for visualizing the reads were generated by igvtools and were visualized on Integrative Genomics Viewer by Broad Institute and Sushi.R (58-60). Enriched peaks for Rvb1/Rvb2 were called using MACS (32). deepTools was used to calculate and plot the enrichment of Rvb1/Rvb2 on genome and selected genes (61). The coverage of reads (BigWig files) was calculated from indexed BAM files using bamCoverage. To compare the enrichment of 2 targets, the matrix was computed from BigWig files of 2 targets using computeMatrix and further presented as a heatmap by plotHeatmap. To visualize Rvb1/Rvb2's enrichment, the matrix was computed from BigWig files of the target and S288C genome annotation as the reference using computeMatrix and further presented by plotProfile. Additionally, BEDtools was used to calculate the coverage of regions of interest and a home-made $\mathrm{R}$ script was generated to analyze and plot the enrichment of Rvb1/Rvb2 on genome and specific gene groups (62).

\section{RNA immunoprecipitation (RIP)}

The RIP protocol was developed based on the protocol from $(8,63) .100 \mathrm{~mL}$ of yeast were grown overnight in SCD medium, until an OD660 around 0.4. $50 \mathrm{~mL}$ of cells were filtered, washed with SC -G media, and resuspended in 50 $\mathrm{mL}$ of prewarmed media and grown at $30^{\circ} \mathrm{C}$ for 15 mins. 50 $\mathrm{mL}$ of pre-starved and 15-minute glucose-starved cell culture was washed and resuspended in $10 \mathrm{~mL}$ of ice-cold PBS buffer, fixed by UV irradiation on a $10-\mathrm{cm}$ petri dish using a Stratalinker $1800(254 \mathrm{~nm}, 9999$ microjoules $\times 100$, $5 \mathrm{~cm}$ from the UV bulb), and harvested. Cells were resuspended in $400 \mu \mathrm{L}$ of ice-cold RIP lysis buffer ( $50 \mathrm{mM}$ Tris $\mathrm{pH}$ 7.5, $100 \mathrm{mM} \mathrm{NaCl}, 1 \% \mathrm{NP}-40,0.5 \%$ SDS, $0.2 \mathrm{mM}$ PMSF, $1 \mathrm{mM}$ DTT, 10U RNase inhibitor from Promega, cOmplete Protease Inhibitor Cocktail from Roche), and lysed by beadbeating (Biospec Products) for 1 minute for 5 times. Brightfield microscopy was used to verify that more than $90 \%$ of cells were lysed. The lysates were centrifuged softly at 1000 $\mathrm{xg}$ at $4^{\circ} \mathrm{C}$ for 10 minutes to remove cell debris and diluted to $500 \mu \mathrm{L}$. Clear lysates were treated with 5U RQ1 DNase and 5U RNase inhibitor (Promega) at $37^{\circ} \mathrm{C}$ for 15 minutes. Then $1 \%$ of the lysate was saved as the input and for Western blotting, respectively. $50 \mu \mathrm{L}$ of IgG-Dynabeads per sample was used. The protocol of preparing the IgG-Dynabeads from Dynabeads M-270 Epoxy (Thermo Fisher) was taken from (53). IgG-Dynabeads were pre-washed 3 times with RIP lysis buffer. The immunoprecipitation (IP) samples were incubated with IgG Dynabeads, rotating at $4^{\circ} \mathrm{C}$ for 4 hours. The IP samples were further washed 6 times by RIP wash buffer (50 mM Tris pH 7.5, $100 \mathrm{mM} \mathrm{NaCl}, 0.1 \% \mathrm{NP}-40$ ) at $4^{\circ} \mathrm{C}$. Samples were later eluted from the beads in $100 \mu \mathrm{L}$ of PK buffer (100 mM Tris pH 7.5, $50 \mathrm{mM} \mathrm{NaCl}, 10 \mathrm{mM}$ EDTA) at $65^{\circ} \mathrm{C}$ for 15 minutes and later the proteins were digested by $10 \mathrm{U}$ Proteinase $\mathrm{K}(\mathrm{NEB})$ at $37^{\circ} \mathrm{C}$ for 30 minutes. Digestion was later activated by incubation with Urea $(210 \mathrm{mg} / \mathrm{mL})$ at $37^{\circ} \mathrm{C}$ for 20 minutes. RNA was extracted using TRIzol reagent (Thermo Fisher) according to the vendor's protocol. RNA was washed twice by $70 \% \mathrm{EtOH}$ and eluted in $10 \mu \mathrm{L}$ of RNase-free water. RNA samples were further digested fully by RQ1 DNase (Promega) in $10 \mu \mathrm{L}$ system and were reverse transcribed by ProtoScript II reverse transcriptase (NEB) (a 1:1 combination of oligo dT18 and random hexamers was used to initiate reverse transcription). The cDNA was investigated by real-time quantitative PCR (RT-qPCR).

\section{Live-cell microscopy and analysis}

Cells were grown to an OD660 to $\sim 0.4$ in SCD medium at $30^{\circ} \mathrm{C}$ and glucose-starved in SC -G medium for 15 and 30 minutes. $100 \mu \mathrm{L}$ of cell culture was loaded onto a 96-well glass-bottom microplate (Cellvis). Cells were imaged using an Eclipse Ti-E microscope (Nikon) with an oil-immersion $63 \mathrm{X}$ objective. Imaging was controlled using NIS-Elements software (Nikon). Imaging analysis was performed on Fiji software.

\section{Nanoluciferase assay and analysis}

The nanoluciferase assay was adapted from methods previously described by (48). Cells were grown to an OD660 to 0.4 in SCD medium at $30^{\circ} \mathrm{C}$ and glucose-starved in SC -G medium for 20 minutes. $90 \mu \mathrm{L}$ of cell culture was loaded onto 
a Cellstar non-transparent white 96-well flat-bottom plate (Sigma-Aldrich). OD660 of cells was taken for each sample. For cells treated with cycloheximide (CHX), CHX was added to a final concentration of $100 \mu \mathrm{g} / \mathrm{mL}$ to stop the translation for 5 minutes. To measure the nanoluciferase signal, $11 \mu \mathrm{L}$ of substrate mix (10 $\mu \mathrm{L}$ of Promega Nano-Glo® Luciferase Assay Buffer, $0.1 \mu \mathrm{L}$ of Promega NanoLuc $\AA$ luciferase substrate and $1 \mu \mathrm{L}$ of $10 \mathrm{mg} / \mathrm{mL}$ CHX) was added and mixed with the samples by pipetting. Measurements were taken immediately after addition of substrate mix by Tecan Infinite Lumi plate reader. To analyze the data, the luciferase level of samples was firstly divided by the OD660 level of the samples. Then the normalized luciferase level of nonCHX-treated sample was further normalized by subtracting the luciferase level of CHX-treated sample.

\section{Western blotting}

The Western blotting protocol was adapted from (64). IP and input samples were mixed with the same volume of $2 \mathrm{X}$ Laemmli buffer (Bio-Rad) and were boiled at $95{ }^{\circ} \mathrm{C}$ for 10 minutes. The samples were then resolved by SDS-PAGE (Bio-Rad), and a rabbit polyclonal antibody specific for calmodulin-binding peptide (A00635-40, GenScript), a Goat anti-Rabbit IgG $(\mathrm{H}+\mathrm{L})$ Secondary Antibody, HRP (Thermo Fisher) and SuperSignal ${ }^{\mathrm{TM}}$ West Femto Maximum Sensitivity Substrate (Thermo Fisher) were used to detect TAP-tagged proteins. The blotting was imaged using a Gel Doc XR+Gel Documentation System (Bio-Rad).

\section{Real-time quantitative PCR (RT-qPCR)}

The RT-qPCR protocol was adapted from (64). RNA was extracted using the MasterPure Yeast RNA Purification Kit (Epicentre). cDNA was prepared using ProtoScript II Reverse Transcriptase (NEB M0368X) with a 1:1 combination of oligodT 18 primers and random hexamers (NEB) according to the manufacturer's instructions. mRNA abundance was determined by qPCR using a home-brew recipe with SYBR Green at a final concentration of $0.5 \mathrm{X}$ (Thermo Fisher S7564). Primers specific for each transcript were described in Supplementary file 2. The mRNA levels were normalized to ACT1 abundance, and the fold change between samples was calculated by a standard $\mathrm{Ct}$ analysis.

\section{Mathematical modeling on the mRNA induction}

The mathematical modeling method was adapted from (35) and performed in Python Jupyter Notebook (https://jupyter.org/). To accurately describe the dynamics of induced mRNA transcription, we used an ordinary differential equation as follows,

$$
\frac{d X}{d t}=\beta-\alpha X
$$

where $\mathrm{X}$ is the mRNA concentration, $\alpha$ is the degradation constant, and $\beta$ is the transcription rate. We assumed that transcription and degradation rates play essential roles in shaping the overall curve of mRNA increase, and these parameters stay constant over the course of induced expression. We then hypothesized that Rvb1/Rvb2 binding to mRNAs could either increase $\beta$ or decrease $\alpha$, leading to greater mRNA abundance than the PP7 control. To observe the effects of varied transcription or degradation rates on the mRNA abundance, we solved the differential equations with different parameters using ODEINT algorithm, and generated time profiles of the mRNA fold in $\log 2$ scale. Solution to the differential equation was expressed as the following function of change in $\mathrm{X}$ with respect to time:

$$
\Delta X(t)=\left(\frac{\beta}{\alpha}-X_{0}\right)\left(1-e^{-\alpha t}\right)
$$

where $X_{0}$ is the mRNA level at $\mathrm{t}=0$, the initial time of mRNA induction. Since the degradation rate was proportional to the mRNA concentration, we expected the curves to have a steady increase, followed by a gradual leveling off where the mRNA concentrations stay constant over time. Closer look at the differential equation showed that at steady state $(\mathrm{dX} / \mathrm{dt}$ $=0$ ), the mRNA concentration is determined by the ratio of $\beta$ to $\alpha$ :

$$
X_{s s}=\frac{\beta}{\alpha}
$$

whereas for the time it takes for the curve to transition into steady state, inversely proportional to the degradation constant, is given by:

$$
T_{\frac{1}{2}}=\ln \frac{2}{\alpha}
$$

Thus, we showed that for $\log 2$ plots, the expected shape of the curves can be altered by varying $\alpha$ and $\beta$. At constant $\alpha$, increasing $\beta$ would only shift the curve up, while at constant $\beta$, increasing $\alpha$ would cause the mRNA abundance to enter steady state more rapidly. Through the comparison between mathematical modeling and experimental data, we could infer the actual effects of Rvb1/Rvb2 binding to mRNA on the transcription and decay rates.

\section{Ribosome profiling}

The ribosome profiling protocol was adpated from (10). Yeast was grown in SCD to an OD660 between 0.3 and 0.4. Then, cells were collected by filtration, resuspended in SC -G medium. After 15 minutes glucose was adding back. CHX was added to a final concentration of $0.1 \mathrm{mg} / \mathrm{mL}$ for $1 \mathrm{~min}$, and cells were then harvested. Cells were pulverized in a PM 100 ball mill (Retsch), and extracts were digested with RNase I followed by the isolation of ribosome-protected fragments by purifying RNA from the monosome fraction of a sucrose gradient. Isolated 28-base sequences were polyadenylated, and reverse transcription was performed using OTi9pA. OTi9pA allowed samples to be multiplexed at subsequent steps. RNA-seq was performed on RNA depleted of rRNA using a yeast Ribo-Zero kit (Epicentre). Samples were multiplexed and sequenced on a HiSeq analyzer (Illumina).

To analyze the ribosomal profiling and RNA-seq sequences, reads were trimmed of the 39 run of poly(A)s and then aligned against $\mathrm{S}$. cerevisiae rRNA sequences using Bowtie sequence aligner (55). Reads that did not align to 
bioRxiv preprint doi: https://doi.org/10.1101/2021.10.17.464753; this version posted October 18, 2021. The copyright holder for this preprint (which was not certified by peer review) is the author/funder, who has granted bioRxiv a license to display the preprint in perpetuity. It is made available under aCC-BY 4.0 International license.

rRNA sequences were aligned against the full S. cerevisiae genome. Reads that had an unambiguous alignment with less than 3 mismatches were used in the measurements of ribosome occupancy and mRNA levels. Since there were many reads mapping to the initiation region (216 bp to120 bp in relation to the AUG), the ribosome occupancy for each mRNA was calculated by taking the total number of ribosome reads (normalized to the total number of aligned reads in reads per million reads (RPM)) in the downstream region (120 bp from the AUG to the end of the ORF) and dividing this by the number of mRNA reads (RPM) in the same region. The ribosome occupancy along the mRNA was calculated by dividing the ribosome read counts at each base pair along the gene by the average number of mRNA reads per base pair for each gene.

\section{Data and materials availability}

ChIP-sequencing reads were deposited at GEO. The raw files and analyzed ChIP-seq enrichment data generated in this study is available at GEO: GSE184473. Further information and requests for data, resources, scripts, and reagents should be directed to and will be fulfilled by the corresponding contact, B.M.Z. (zid@ucsd.edu).

\section{Acknowledgements}

We would like to thank the Zid lab especially Anna R. Guzikowski and Tatsuhisa Tsuboi for helpful feedback on this manuscript. We thank Toshio Tsukiyama for sharing the LacI-Flag and pUC-TalO8 plasmids. This work was in part supported by the National Institutes of Health R35GM128798 (to BMZ).

\section{Author contributions}

Y.S.C., Conceptualization, Data curation, Formal analysis, Investigation, Methodology, Visualization, Writing - original draft, Writing - review and editing; S.T., Methodology - CoTrIP, ChIP-seq, Investigation - CoTrIP; V.H., Investigation - Strain construction, Writing - review and editing; F.X., Mathematical modeling, Writing - review and editing; J.J.M., Methodology - CoTrIP, ChIP-seq, Investigation - CoTrIP; J.R.Y., Supervision, Resources, Methodology CoTrIP; B.M.Z., Conceptualization, Data curation, Formal analysis, Funding acquisition, Investigation, Methodology, Project administration, Resources, Supervision, Writing original draft, Writing - review and editing.

\section{References}

1. Amar J. Majmundar, Waihay J. Wong, and M. Celeste Simon. Hypoxia-Inducible Factors and the Response to Hypoxic Stress, oct 2010. ISSN 10972765.

2. Klaus Richter, Martin Haslbeck, and Johannes Buchner. The Heat Shock Response: Life on the Verge of Death, oct 2010. ISSN 10972765.

3. Eulélia De Nadal, Gustav Ammerer, and Francesc Posas. Controlling gene expression in response to stress, dec 2011. ISSN 14710056.

4. Gal Haimovich, Mordechai Choder, Robert H. Singer, and Tatjana Trcek. The fate of the messenger is pre-determined: A new model for regulation of gene expression. Biochimica et Biophysica Acta (BBA) - Gene Regulatory Mechanisms, 1829(6-7):643-653, jun 2013. ISSN 18749399. doi: 10.1016/j.bbagrm.2013.01.004.

5. Almog Bregman, Moran Avraham-Kelbert, Oren Barkai, Lea Duek, Adi Guterman, and Mordechai Choder. Promoter Elements Regulate Cytoplasmic mRNA Decay. Cell, 147 (7):1473-1483, dec 2011. ISSN 00928674. doi: 10.1016/j.cell.2011.12.005.
6. Tatjana Trcek, Daniel R. Larson, Alberto Moldón, Charles C. Query, and Robert H. Singer. Single-Molecule mRNA Decay Measurements Reveal Promoter- Regulated mRNA Stability in Yeast. Cell, 147(7):1484-1497, dec 2011. ISSN 00928674. doi: 10.1016/j.cell.2011.11. 051.

7. Maria Vera, Bibhusita Pani, Lowri A Griffiths, Christian Muchardt, Catherine M Abbott, Robert $\mathrm{H}$ Singer, and Evgeny Nudler. The translation elongation factor eEF1A1 couples transcription to translation during heat shock response. eLife, 3, sep 2014. ISSN 2050084X. doi: 10.7554/elife.03164.

8. Gesa Zander, Alexandra Hackmann, Lysann Bender, Daniel Becker, Thomas Lingner, Gabriela Salinas, and Heike Krebber. mRNA quality control is bypassed for immediate export of stress-responsive transcripts. Nature, 540(7634):593-596, dec 2016. ISSN 14764687. doi: 10.1038/nature20572.

9. Anna R. Guzikowski, Yang S. Chen, and Brian M. Zid. Stress-induced mRNP granules: Form and function of processing bodies and stress granules. Wiley Interdisciplinary Reviews: RNA, 10(3):e1524, may 2019. ISSN 1757-7012. doi: 10.1002/wrna.1524.

10. Brian M. Zid and Erin K. O'Shea. Promoter sequences direct cytoplasmic localization and translation of mRNAs during starvation in yeast. Nature, 514(7520):117-121, oct 2014. ISSN 14764687. doi: 10.1038/nature13578.

11. David S.W. Protter and Roy Parker. Principles and Properties of Stress Granules, sep 2016. ISSN 18793088.

12. Ji Young Youn, Boris J.A. Dyakov, Jianping Zhang, James D.R. Knight, Robert M Vernon, Julie D Forman-Kay, and Anne Claude Gingras. Properties of Stress Granule and P-Body Proteomes, 2019. ISSN 10974164.

13. Kathleen M. Attwood, Aaron Robichaud, Lauren P. Westhaver, Elizabeth L. Castle, David M. Brandman, Aruna D. Balgi, Michel Roberge, Patricia Colp, Sidney Croul, Inhwa Kim, Craig McCormick, Jennifer A. Corcoran, and Adrienne Weeks. Raloxifene prevents stress granule dissolution, impairs translational control and promotes cell death during hypoxia in glioblastoma cells. Cell Death and Disease, 11(11):1-18, nov 2020. ISSN 20414889. doi: 10.1038/s41419-020-03159-5.

14. Pavel Ivanov, Nancy Kedersha, and Paul Anderson. Stress granules and processing bodies in translational control. Cold Spring Harbor Perspectives in Biology, 11(5):a032813, may 2019. ISSN 19430264. doi: 10.1101/cshperspect.a032813.

15. N. Kedersha and P. Anderson. Stress granules: sites of mRNA triage that regulate mRNA stability and translatability. Biochemical Society Transactions, 2002. ISSN 0300-5127. doi: $10.1042 /$ bst0300963.

16. Pabitra K. Sahoo, Seung Joon Lee, Poonam B. Jaiswal, Stefanie Alber, Amar N. Kar, Sharmina Miller-Randolph, Elizabeth E. Taylor, Terika Smith, Bhagat Singh, Tammy Szu Yu Ho, Anatoly Urisman, Shreya Chand, Edsel A. Pena, Alma L. Burlingame, Clifford J. Woolf, Mike Fainzilber, Arthur W. English, and Jeffery L. Twiss. Axonal G3BP1 stress granule protein limits axonal mRNA translation and nerve regeneration. Nature Communications, 9(1): 1-14, dec 2018. ISSN 20411723. doi: 10.1038/s41467-018-05647-x.

17. Patrik Eickhoff and Alessandro Costa. Escorting Client Proteins to the Hsp90 Molecular Chaperone, jul 2017. ISSN 18784186.

18. Jennifer Huen, Yoshito Kakihara, Francisca Ugwu, Kevin L.Y. Cheung, Joaquin Ortega, and Walid A. Houry. Rvb1-Rvb2: Essential ATP-dependent helicases for critical complexes. In Biochemistry and Cell Biology, volume 88, pages 29-40, feb 2010. doi: 10.1139/O09-122.

19. S Jha and A Dutta. RVB1/RVB2: running rings around molecular biology. Mol Cell, 34(5): 521-533, 2009. doi: 10.1016/j.molcel.2009.05.016.

20. Nardin Nano and Walid A. Houry. Chaperone-like activity of the AAApproteins RVb1 and RVb2 in the assembly of various complexes, 2013. ISSN 14712970.

21. Alexandr Paci, Xiao Hu Liu, Hao Huang, Abelyn Lim, Walid A Houry, and Rongmin Zhao. The stability of the small nucleolar ribonucleoprotein (snoRNP) assembly protein Pih1 in Saccharomyces cerevisiae is modulated by its $\mathrm{C}$ terminus. Journal of Biological Chemistry, 287(52):43205-43214, 2012. ISSN 00219258. doi: 10.1074/jbc.M112.408849.

22. Thiago V. Seraphim, Nardin Nano, Yiu Wing Sunny Cheung, Siripat Aluksanasuwan, Carolina Colleti, Yu-Qian Mao, Vaibhav Bhandari, Gavin Young, Larissa Höll, Sadhna Phanse, Yuliya Gordiyenko, Daniel R. Southworth, Carol V. Robinson, Visith Thongboonkerd, Lisandra M. Gava, Júlio C. Borges, Mohan Babu, Leandro R.S. Barbosa, Carlos H.I. Ramos, Philipp Kukura, and Walid A. Houry. Assembly principles of the human R2TP chaperone complex reveal the presence of R2T and R2P complexes. Structure, 0(0), sep 2021. ISSN 09692126. doi: 10.1016/j.str.2021.08.002.

23. Shaoxiong Tian, Ge Yu, Huan He, Yu Zhao, Peilu Liu, Alan G. Marshall, Borries Demeler, Scott M. Stagg, and Hong Li. Pih1p-Tah1p Puts a Lid on Hexameric AAA+ ATPases Rvb1/2p. Structure, 25(10):1519-1529.e4, oct 2017. ISSN 18784186. doi: 10.1016/j. str.2017.08.002.

24. S Jain, J R Wheeler, R W Walters, A Agrawal, A Barsic, and R Parker. ATPase-Modulated Stress Granules Contain a Diverse Proteome and Substructure. Cell, 164(3):487-498, 2016. doi: 10.1016/j.cell.2015.12.038.

25. Arjun Narayanan, Anatoli Meriin, J Owen Andrews, Jan-Hendrik Spille, Michael Y Sherman, and Ibrahim I Cisse. A first order phase transition mechanism underlies protein aggregation in mammalian cells. eLife, 8, feb 2019. ISSN 2050-084X. doi: 10.7554/elife.39695.

26. Nava Zaarur, Xiaobin Xu, Patrick Lestienne, Anatoli B Meriin, Mark McComb, Catherine E Costello, Gary P Newnam, Rakhee Ganti, Nina V Romanova, Maruda Shanmugasundaram, Sara Tn Silva, Tiago M Bandeiras, Pedro M Matias, Kirill S Lobachev, Igor K Lednev, Yury O Chernoff, and Michael Y Sherman. RuvbL1 and RuvbL2 enhance aggresome formation and disaggregate amyloid fibrils. The EMBO Journal, 34(18):2363-2382, sep 2015. ISSN 0261-4189. doi: 10.15252/embj.201591245.

27. Xin Mu, Yajing Fu, Yiping Zhu, Xinlu Wang, Yifang Xuan, Hong Shang, Stephen P. Goff, and Guangxia Gao. HIV-1 Exploits the Host Factor RuvB-like 2 to Balance Viral Protein Expression. Cell Host Microbe, 18(2):233-242, aug 2015. ISSN 19346069. doi: 10.1016/j. chom.2015.06.018.

28. Ashwin Unnikrishnan, Philip R. Gafken, and Toshio Tsukiyama. Dynamic changes in histone acetylation regulate origins of DNA replication. Nature Structural and Molecular Biology, 17 (4):430-437, apr 2010. ISSN 15459993. doi: 10.1038/nsmb.1780.

29. Ashwin Unnikrishnan, Bungo Akiyoshi, Sue Biggins, and Toshio Tsukiyama. An efficient purification system for native minichromosome from saccharomyces cerevisiae. Methods in 
bioRxiv preprint doi: https://doi.org/10.1101/2021.10.17.464753; this version posted October 18, 2021. The copyright holder for this preprint (which was not certified by peer review) is the author/funder, who has granted bioRxiv a license to display the preprint in perpetuity. It is made available under aCC-BY 4.0 International license.

Molecular Biology, 833:115-123, 2012. ISSN 10643745. doi: 10.1007/978-1-61779-477-3_ 8 .

30. D Mellacheruvu, Z Wright, A L Couzens, J P Lambert, N A St-Denis, T Li, Y V Miteva, S Hauri, M E Sardiu, T Y Low, V A Halim, R D Bagshaw, N C Hubner, A Al-Hakim, A Bouchard, D Faubert, D Fermin, W H Dunham, M Goudreault, Z Y Lin, B G Badillo, T Pawson, D Durocher, B Coulombe, R Aebersold, G Superti-Furga, J Colinge, A J Heck, H Choi, M Gstaiger, S Mohammed, I M Cristea, K L Bennett, M P Washburn, B Raught, R M Ewing, A C Gingras, and A I Nesvizhskii. The CRAPome: a contaminant repository for affinity purification-mass spectrometry data. Nat Methods, 10(8):730-736, 2013. doi: 10.1038/nmeth.2557.

31. Coral Y. Zhou, Caitlin I. Stoddard, Jonathan B. Johnston, Michael J. Trnka, Ignacia Echeverria, Eugene Palovcak, Andrej Sali, Alma L. Burlingame, Yifan Cheng, and Geeta J. Narlikar. Regulation of Rvb1/Rvb2 by a Domain within the INO80 Chromatin Remodeling Complex Implicates the Yeast Rvbs as Protein Assembly Chaperones. Cell Reports, 19(10):20332044, jun 2017. ISSN 22111247. doi: 10.1016/j.celrep.2017.05.029.

32. Yong Zhang, Tao Liu, Clifford A. Meyer, Jérôme Eeckhoute, David S. Johnson, Bradley E. Bernstein, Chad Nussbaum, Richard M. Myers, Myles Brown, Wei Li, and X. Shirley Shirley. Model-based analysis of ChIP-Seq (MACS). Genome Biology, 9(9):1-9, sep 2008. ISSN 14747596. doi: 10.1186/gb-2008-9-9-r137.

33. Natsuko Izumi, Akio Yamashita, and Shigeo Ohno. Integrated regulation of PIKK-mediated stress responses by AAA+ proteins RUVBL1 and RUVBL2, 2012. ISSN 19491042.

34. Francis Lim and David S. Peabody. RNA recognition site of PP7 coat protein, oct 2002. ISSN 03051048.

35. Ran Elkon, Eitan Zlotorynski, Karen I Zeller, and Reuven Agami. Major role for mRNA stability in shaping the kinetics of gene induction. BMC Genomics, 11(1):1-8, apr 2010. ISSN 14712164. doi: 10.1186/1471-2164-11-259.

36. Ivana Horvathova, Franka Voigt, Anna V. Kotrys, Yinxiu Zhan, Caroline G. Artus-Revel, Jan Eglinger, Michael B. Stadler, Luca Giorgetti, and Jeffrey A. Chao. The Dynamics of mRNA Turnover Revealed by Single-Molecule Imaging in Single Cells. Molecular Cell, 68(3):615625.e9, nov 2017. ISSN 10974164. doi: 10.1016/j.molcel.2017.09.030.

37. Sethuramasundaram Pitchiaya, Marcio D.A. Mourao, Ameya P. Jalihal, Lanbo Xiao, Xia Jiang, Arul M. Chinnaiyan, Santiago Schnell, and Nils G. Walter. Dynamic Recruitment of Single RNAs to Processing Bodies Depends on RNA Functionality. Molecular Cell, 74(3): 521-533.e6, may 2019. ISSN 10974164. doi: 10.1016/j.molcel.2019.03.001.

38. Stefan Schutz, Erik R. Noldeke, and Remco Sprangers. A synergistic network of interactions promotes the formation of in vitro processing bodies and protects mRNA against decapping. Nucleic Acids Research, 45(11):6911-6922, jun 2017. ISSN 13624962. doi: 10.1093/nar/ gkx353.

39. Yanfei Jiang, Zohreh AkhavanAghdam, Yutian Li, Brian M. Zid, and Nan Hao. A protein kinase A-regulated network encodes short- And long-lived cellular memories. Science Signaling, 13(632), may 2020. ISSN 19379145. doi: 10.1126/scisignal.aaw8905.

40. Zophonías O. Jónsson, Suman K Dhar, Geeta J Narlikar, Roy Auty, Nikhil Wagle, David Pellman, Richard E Pratt, Robert Kingston, and Anindya Dutta. Rvb1p and Rvb2p are Essential Components of a Chromatin Remodeling Complex that Regulates Transcription of over 5\% of Yeast Genes. Journal of Biological Chemistry, 276(19):16279-16288, 2001. ISSN 00219258. doi: 10.1074/jbc.M011523200.

41. Surabhi Chowdhary, Amoldeep S. Kainth, and David S Gross. Heat Shock Protein Genes Undergo dynamic alteration in their Three-Dimensional Structure and Genome organization in response to thermal Stress. Molecular and cellular biology, 37(24):1-22, 2017. ISSN 1098-5549. doi: 10.1128/MCB.00292-17.

42. David Pincus, Jayamani Anandhakumar, Prathapan Thiru, Michael J. Guertin, Alexander M. Erkine, and David S. Gross. Genetic and epigenetic determinants establish a continuum of Hsf1 occupancy and activity across the yeast genome. Molecular Biology of the Cell, pages mbc.E18-06-0353, oct 2018. ISSN 1059-1524. doi: 10.1091/mbc.E18-06-0353.

43. Aude Grigoletto, Patrick Lestienne, and Jean Rosenbaum. The multifaceted proteins Reptin and Pontin as major players in cancer, 2011. ISSN 0304419X.

44. Johannes C. Lauscher, Sefer Elezkurtaj, Sonja Dullat, Sybille Lipka, Jörn Grone, Heinz J. Buhr, Otmar Huber, and Martin Kruschewski. Increased Pontin expression is a potential predictor for outcome in sporadic colorectal carcinoma. Oncology Reports, 28(5):16191624, 2012. ISSN 1021335X. doi: 10.3892/or.2012.1968.

45. Derrick Lin, Brian Lin, Haymanti Bhanot, Rozenn Riou, Nicholas B. Abt, Jayaraj Rajagopal, and Srinivas Vinod Saladi. RUVBL1 is an amplified epigenetic factor promoting proliferation and inhibiting differentiation program in head and neck squamous cancers. Oral Oncology, 111(June):104930, 2020. ISSN 18790593. doi: 10.1016/j.oraloncology.2020.104930.

46. Sidae Lee, Wendell A. Lim, and Kurt S. Thorn. Improved Blue, Green, and Red Fluorescent Protein Tagging Vectors for S. cerevisiae. PLOS ONE, 8(7):e67902, jul 2013. ISSN 19326203. doi: 10.1371/journal.pone.0067902.

47. Mark S Longtine, Amos McKenzie III, Douglas J Demarini, Nirav G Shah, Achim Wach, Arndt Brachat, Peter Philippsen, and John R Pringle. Additional modules for versatile and economical PCR-based gene deletion and modification in Saccharomyces cerevisiae. Yeast, 14(10):953-961, 1998.

48. Anna E. Masser, Ganapathi Kandasamy, Jayasankar Mohanakrishnan Kaimal, and Claes Andréasson. Luciferase NanoLuc as a reporter for gene expression and protein levels in Saccharomyces cerevisiae. Yeast, 33(5):191-200, may 2016. ISSN 10970061. doi: 10. 1002/yea.3155.

49. W. Hayes McDonald, David L. Tabb, Rovshan G. Sadygov, Michael J. MacCoss, John Venable, Johannes Graumann, Jeff R. Johnson, Daniel Cociorva, and John R. Yates. MS1, MS2, and SQT - Three unified, compact, and easily parsed file formats for the storage of shotgun proteomic spectra and identifications. Rapid Communications in Mass Spectrometry, 18(18):2162-2168, sep 2004. ISSN 09514198. doi: 10.1002/rcm.1603.

50. Junmin Peng, Daniel Schwartz, Joshua E Elias, Carson C Thoreen, Dongmei Cheng, Gerald Marsischky, Jeroen Roelofs, Daniel Finley, and Steven P Gygi. A proteomics approach to understanding protein ubiquitination. Nature Biotechnology, 21(8):921-926, jul 2003. ISSN 10870156. doi: 10.1038/nbt849.

51. T. Xu, S. K. Park, J. D. Venable, J. A. Wohlschlegel, J. K. Diedrich, D. Cociorva, B. Lu, L. Liao, J. Hewel, X. Han, C. C.L. Wong, B. Fonslow, C. Delahunty, Y. Gao, H. Shah, and
J. R. Yates. ProLuCID: An improved SEQUEST-like algorithm with enhanced sensitivity and specificity. Journal of Proteomics, 129:16-24, nov 2015. ISSN 18767737. doi: 10.1016/j. jprot.2015.07.001.

52. Melanie Grably and David Engelberg. A detailed protocol for chromatin immunoprecipitation in the yeast Saccharomyces cerevisiae. Methods in molecular biology (Clifton, N.J.), 638: 211-224, 2010. ISSN 19406029. doi: 10.1007/978-1-60761-611-5_16.

53. Xiyan Li. Rabbit IgG Conjugation to Dynabeads. BIO-PROTOCOL, 1(9), 2011. ISSN 23318325. doi: 10.21769/bioprotoc.63.

54. Simon Anders. Babraham Bioinformatics - FastQC A Quality Control tool for High Throughput Sequence Data, 2010.

55. Ben Langmead and Steven $L$ Salzberg. Fast gapped-read alignment with Bowtie 2. Nature Methods, 9(4):357-359, mar 2012. ISSN 15487091. doi: 10.1038/nmeth.1923.

56. Heng Li, Bob Handsaker, Alec Wysoker, Tim Fennell, Jue Ruan, Nils Homer, Gabor Marth, Goncalo Abecasis, and Richard Durbin. The Sequence Alignment/Map format and SAMtools. Bioinformatics, 25(16):2078-2079, aug 2009. ISSN 13674803. doi: 10.1093/bioinformatics/btp352.

57. Broad Institute. Picard Tools - By Broad Institute, 2009

58. Douglas H. Phanstiel, Alan P. Boyle, Carlos L. Araya, and Michael P. Snyder. Sushi.R: Flexible, quantitative and integrative genomic visualizations for publication-quality multipanel figures. Bioinformatics, 30(19):2808-2810, oct 2014. ISSN 14602059. doi: 10.1093/bioinformatics/btu379.

59. James T Robinson, Helga Thorvaldsdóttir, Wendy Winckler, Mitchell Guttman, Eric S Lander, Gad Getz, and Jill P Mesirov. Integrative genomics viewer, jan 2011. ISSN 10870156.

60. Helga Thorvaldsdóttir, James T. Robinson, and Jill P. Mesirov. Integrative Genomics Viewer (IGV): High-performance genomics data visualization and exploration. Briefings in Bioinformatics, 14(2):178-192, mar 2013. ISSN 14675463. doi: 10.1093/bib/bbs017.

61. Fidel Ramírez, Devon P Ryan, Björn Grüning, Vivek Bhardwaj, Fabian Kilpert, Andreas $S$ Richter, Steffen Heyne, Friederike Dündar, and Thomas Manke. deepTools2: a next generation web server for deep-sequencing data analysis. Nucleic acids research, 44(W1): W160-W165, jul 2016. ISSN 13624962. doi: 10.1093/nar/gkw257.

62. Aaron R. Quinlan and Ira M. Hall. BEDTools: A flexible suite of utilities for comparing genomic features. Bioinformatics, 26(6):841-842, mar 2010. ISSN 13674803. doi: 10. 1093/bioinformatics/btq033.

63. E L Van Nostrand, G A Pratt, A A Shishkin, C Gelboin-Burkhart, M Y Fang, B Sundararaman, S M Blue, T B Nguyen, C Surka, K Elkins, R Stanton, F Rigo, M Guttman, and G W Yeo. Robust transcriptome-wide discovery of RNA-binding protein binding sites with enhanced CLIP (eCLIP). Nat Methods, 13(6):508-514, 2016. doi: 10.1038/nmeth.3810.

64. Tatsuhisa Tsuboi, Matheus P. Viana, Fan Xu, Jingwen Yu, Raghav Chanchani, Ximena G. Arceo, Evelina Tutucci, Joonhyuk Choi, Yang S. Chen, Robert H. Singer, Susanne M. Rafelski, and Brian M. Zid. Mitochondrial volume fraction and translation duration impact mitochondrial mRNA localization and protein synthesis. eLife, 9:1-24, aug 2020. ISSN 2050084X. doi: 10.7554/ELIFE.57814. 